INTERNATIONAL JOURNAL FOR NUMERICAL METHODS IN ENGINEERING

Int. J. Numer. Meth. Engng 2000; 00:1-6

Prepared using nmeauth.cls [Version: 2002/09/18 v2.02]

\title{
Extended digital image correlation with crack shape optimization
}

\author{
Julien Réthoré, ${ }^{1}$ François Hild, ${ }^{1 *}$, and Stéphane Roux ${ }^{2}$ \\ ${ }^{1}$ Laboratoire de Mécanique et Technologie (LMT-Cachan) \\ ENS-Cachan, CNRS UMR 8535, Université Paris VI, Cachan, France \\ ${ }^{2}$ Surface du Verre et Interfaces \\ Unité Mixte de Recherche CNRS/Saint-Gobain, Aubervilliers, France
}

SUMMARY

The methodology of eXtended Finite Element Method is applied to the measurement of displacements through digital image correlation. An algorithm, initially based on a finite element decomposition of displacement fields, is extended to benefit from discontinuity and singular enrichments over a suited subset of elements. This allows one to measure irregular displacements encountered, say, in cracked solids, as demonstrated both on artificial examples and experimental case studies. Moreover, an optimization strategy for the support of the discontinuity enables one to adjust the crack path configuration to reduce the residual mismatch, and hence to be tailored automatically to a wavy or irregular crack path. Copyright (c) 2000 John Wiley \& Sons, Ltd.

\footnotetext{
*Correspondence to: François Hild, Laboratoire de Mécanique et de Technologie (LMT-Cachan), ENS de Cachan, 61 Avenue du Président Wilson, F-94235 Cachan, France, hild@lmt.ens-cachan.fr
}

Contract/grant sponsor: CETIM Foundation; contract/grant number: This work was funded by the CETIM Foundation grant entitled PROPAVANFIS: "Advanced methods for the experimental and numerical analyses of crack propagations under complex loadings."

Received 
KEY WORDS: X-FEM, image correlation, crack front, displacement field measurement

\section{INTRODUCTION}

Digital image correlation is now a quite well established tool for measuring displacement fields [22]. This technique is all the more interesting when the displacement field is heterogeneous, for example when discontinuities exist in the specimen [11]. Another appealing feature is that the conservation of the optical flow, which is the underlying identification principle, may be discretized over the entire area of the image by using finite element shape functions [3]. Consequently, the discretization scheme used during measurements and simulations may be the same. This property unifies the measurement-identification-simulation loop thereby reducing errors induced by intermediate projection steps. However, because of the very nature of the measurement procedure (i.e., an ill-posed problem in the Hadamard sense), a finite element discretization does not possess the well-known convergence properties it shows as a partial differential equation solving technique. The standard displacement uncertainty decreases when the element size increases. Being less flexible to follow the actual displacement field, it is also less prone to noise. The amount of data to constrain the nodal unknowns of the displacement field increases with the element size. Therefore, measurements involve intrinsically a compromise between spatial resolution (i.e., element size) and displacement uncertainty [3].

To illustrate quantitatively this phenomenon, let us consider the analysis of the three different situations shown schematically in Figure 1. First, a rigid body translation of 0.5 pixel along both directions is artificially applied to a given picture. A cubic spline algorithm is used to 
interpolate the gray level value in the "transformed" picture. The analysis of the displacement is performed using a finite element Q4 basis for elements whose size is $\ell \times \ell$ pixels. The quality of the determination, here called "uncertainty," is evaluated through the standard deviation of the difference between the known and measured displacement fields. Figure 2 illustrates the compromise mentioned earlier, namely, the larger the element size, the smaller the standard displacement uncertainty. The very abrupt decrease in uncertainty with the element size is a result of the reduction in noise sensitivity with the number of pixels. Second, a discontinuous translation (i.e., the left part of the picture is fixed, and the right part is translated by 0.5 pixel along both directions) is applied. The displacement uncertainty now increases with the element size. Note however that the overall level is still less than $5 \times 10^{-2}$ pixel for elements of size 64 pixels. Third, the displacement field corresponding to a mixed mode I and II crack with a stress intensity factor such that the maximum displacement jump is 1 pixel in each direction is created. In this last case, the uncertainty level is of the order of 0.1 pixel for elements of size 64 pixels. The reason for such a degradation of the uncertainty as the element size increases in the two last cases is due to the fact that the searched displacement field is continuous by hypothesis, and thus cannot accurately describe the displacement jump. The larger the elements, the wider the region that suffers from this approximation. In contrast, a uniform displacement (first case) lies inside the chosen displacement space, and thus only the noise reduction effect is felt. In order to prove this point, Figure 2 also shows the unavoidable contribution to the uncertainty due to the nodal projection of the imposed discontinuous displacement onto the basis of chosen functions (Q4 shape functions). For discontinuous translations, the uncertainty induced by the correlation is greater than that given by nodal projection. However, the dominant effect is the latter, i.e., it is mainly related to the kinematic 
hypothesis made at the measurement stage. For the crack tip field, it is to be mentioned that a nodal projection is not the best choice to minimize the error made on the displacement map (not only on the nodal values). Using an appropriate projection algorithm (least-squares for example), the incompressible contribution of the Q4 shape function should be less than what is obtained by correlation. These results show that, even though reasonable displacement uncertainties are observed, suited procedures may yield better results.

Confronted to a similar situation, various remedies have been proposed within the framework of finite element simulations. Among those, the eXtended Finite Element Method (X-FEM) is able to reveal heterogeneous displacement fields with arbitrary discontinuities without remeshing $[4,12,2]$. Enriched functions are added to the initial finite element shape functions exploiting their partition of unity property [1]. X-FEM usually allows one to use coarser meshes for the same accuracy in the numerical solution. This property is all the more interesting in the context of digital image correlation for which coarser mesh usually means lower measurement uncertainties. Therefore, the use of an enriched interpolation shall bring less error, less uncertainty, and hopefully a better spatial resolution at the same time. This paper is concerned by the extension " $a$ la $X-F E M$ " of a recent Q4 correlation technique [3].

The paper is organized as follows. First, general principles of image correlation are recalled. Then, the computational details of the Q4 as well as the eXtended Q4-algorithms are presented. The performance of the approach is illustrated with an uncertainty analysis and an experimental case study. Since real cracks are usually not straight, an algorithm is then proposed to determine the crack geometry. It is based on a two-scale multi-field minimization of the correlation error due to non-matching between the real crack path and the assumed one used in the correlation algorithm. Artificial and experimental examples validate the proposed 
technique.

\section{PRINCIPLES OF IMAGE CORRELATION}

Two digital (gray level) images corresponding respectively to a reference, $f(\mathbf{x})$, and a deformed, $g(\mathbf{x})$, state are related by the "passive" advection of the local texture in the displacement field

$\mathbf{u}$

$$
g(\mathbf{x})=f(\mathbf{x}+\mathbf{u}(\mathbf{x}))
$$

This equation corresponds to the local form of the so-called optical flow conservation principle. From the knowledge of $f$ and $g$, the problem consists in estimating the displacement $\mathbf{u}$ as accurately as possible.

\subsection{General framework}

The measurement of the displacement is an ill-posed problem. The displacement is only measurable along the direction of the intensity gradient. Consequently, additional hypotheses have to be proposed to solve the problem. It is simplified by considering incrementally linearized tangent problems. Equation (1) is rewritten by assuming that the reference image is differentiable to yield the local square residual

$$
\Phi^{2}(\mathbf{x})=[\mathbf{u}(\mathbf{x}) \cdot \nabla f(\mathbf{x})+f(\mathbf{x})-g(\mathbf{x})]^{2}
$$

To estimate $\mathbf{u}$, the quadratic residual $\Phi^{2}$ is integrated over the studied domain $\Omega$

$$
\eta^{2}=\iint_{\Omega}[\mathbf{u}(\mathbf{x}) \cdot \nabla f(\mathbf{x})+f(\mathbf{x})-g(\mathbf{x})]^{2} \mathrm{~d} \mathbf{x}
$$

and subsequently minimized. The displacement field is decomposed over a set of functions $\boldsymbol{\Psi}_{n}(\mathbf{x})$ to regularize the measurement problem. Each component of the displacement field is 
treated in a similar manner, and thus only scalar shape functions $\psi_{n}(\mathbf{x})$ are introduced

$$
\mathbf{u}(\mathbf{x})=\sum_{\alpha, n} a_{\alpha n} \psi_{n}(\mathbf{x}) \mathbf{e}_{\alpha}
$$

where $\mathbf{e}_{\alpha}$ are elementary unit vectors along each space dimension $\alpha$ and $a_{\alpha n}$ the corresponding amplitudes. The objective function is thus expressed as

$$
\eta^{2}=\iint_{\Omega}\left[\sum_{\alpha, n} a_{\alpha n} \psi_{n}(\mathbf{x}) \nabla f(\mathbf{x}) \cdot \mathbf{e}_{\alpha}+f(\mathbf{x})-g(\mathbf{x})\right]^{2} \mathrm{~d} \mathbf{x}
$$

and hence its minimization leads to a linear system

$$
\begin{array}{r}
\sum_{\beta, m} a_{\beta m} \iint_{\Omega}\left[\psi_{m}(\mathbf{x}) \psi_{n}(\mathbf{x}) \partial_{\alpha} f(\mathbf{x}) \partial_{\beta} f(\mathbf{x})\right] \mathrm{d} \mathbf{x}= \\
=\iint[g(\mathbf{x})-f(\mathbf{x})] \psi_{n}(\mathbf{x}) \partial_{\alpha} f(\mathbf{x}) \mathrm{d} \mathbf{x}
\end{array}
$$

that is written in a compact form as

$$
\mathrm{Ma}=\mathbf{b}
$$

where $\partial_{\alpha} f=\nabla f . \mathbf{e}_{\alpha}$ denotes the directional derivative, the matrix $\mathbf{M}$ and the vector $\mathbf{b}$ are directly read from Eq. (6)

$$
M_{\alpha n \beta m}=\iint_{\Omega}\left[\psi_{m}(\mathbf{x}) \psi_{n}(\mathbf{x}) \partial_{\alpha} f(\mathbf{x}) \partial_{\beta} f(\mathbf{x})\right] \mathrm{d} \mathbf{x}
$$

and

$$
b_{\alpha n}=\iint_{\Omega}[g(\mathbf{x})-f(\mathbf{x})] \psi_{n}(\mathbf{x}) \partial_{\alpha} f(\mathbf{x}) \mathrm{d} \mathbf{x}
$$

The present development is similar to a Rayleigh-Ritz procedure frequently used in elastic analyzes. The only difference corresponds to the fact that the variational formulation is associated with a weak form of the (linearized) conservation of optical flow and not the principle of virtual work. However, the linearization in this case is problematic because the irregular texture of the images may lead to multiple local minima of the residual. A hierarchical multiscale approach is used to achieve convergence to the global minimum of the residual $[10,3]$. 
This procedure is similar to a Full Approximation Scheme multi-grid algorithm with only one step down from the coarsest grid to the finest one. Three grid levels are usually considered in the presented applications.

\subsection{Q4-Digital Image Correlation}

A large variety of functions $\Psi$ may be considered. Among them, finite element shape functions [3] are particularly attractive because of the natural interface they provide between the measurement of the displacement field and a numerical modeling of it based on a constitutive equation. Whatever the strategy chosen for the identification of the constitutive parameters, choosing an identical kinematic description suppresses spurious numerical noise at the comparison step. Moreover, since the image is naturally partitioned into pixels, it is appropriate to choose a square or rectangular shape for each element. This leads us to the choice of Q4-finite elements as the simplest basis. Each element is mapped onto the square $[-1,1]^{2}$, where the four basic (i.e., P1) functions are $1 / 4(1 \pm x)(1 \pm y)$ in a local $(x, y)$ frame. The displacement decomposition (4) is therefore particularized to account for the previous shape functions of a finite element discretization. Each component of the displacement field is treated in a similar way, and thus only scalar shape functions $N_{n}(\mathbf{x})$ are introduced to interpolate the displacement $\mathbf{u}^{e}(\mathbf{x})$ in an element $\Omega_{e}$

$$
\mathbf{u}^{e}(\mathbf{x})=\sum_{n=1}^{n_{e}} \sum_{\alpha} a_{\alpha n} N_{n}(\mathbf{x}) \mathbf{e}_{\alpha}
$$

where $n_{e}$ is the number of nodes (here $n_{e}=4$ ), and $a_{\alpha n}$ the unknown nodal displacements. The objective function is recast as

$$
\eta^{2}=\sum_{e} \iint_{\Omega_{e}}\left[\sum_{\alpha, n} a_{\alpha n} N_{n}(\mathbf{x}) \nabla f(\mathbf{x}) \cdot \mathbf{e}_{\alpha}+f(\mathbf{x})-g(\mathbf{x})\right]^{2} \mathrm{~d} \mathbf{x}
$$

Copyright (C) 2000 John Wiley \& Sons, Ltd.

Int. J. Numer. Meth. Engng 2000; 00:1-6

Prepared using nmeauth.cls 
and hence its minimization leads to a linear system (6) in which the matrix $\mathbf{M}$ is obtained from the assembly of the elementary matrices $\mathbf{M}^{e}$ whose components read

$$
M_{\alpha n \beta m}^{e}=\iint_{\Omega_{e}}\left[N_{m}(\mathbf{x}) N_{n}(\mathbf{x}) \partial_{\alpha} f(\mathbf{x}) \partial_{\beta} f(\mathbf{x})\right] \mathrm{d} \mathbf{x}
$$

In practice, a symmetrization is performed by using $\partial_{\alpha} \frac{f+g}{2} \partial_{\beta} \frac{f+g}{2}$ instead of $\partial_{\alpha} f \partial_{\beta} f$. The vector $\mathbf{b}$ corresponds to the assembly of the elementary vectors $\mathbf{b}^{e}$ such that

$$
b_{\alpha n}^{e}=\iint_{\Omega_{e}}[g(\mathbf{x})-f(\mathbf{x})] N_{n}(\mathbf{x}) \partial_{\alpha} f(\mathbf{x}) \mathrm{d} \mathbf{x}
$$

The same symmetrization, i.e., substituting $\partial_{\alpha}(f+g) / 2$ to $\partial_{\alpha} f$, is considered. Thus it is straightforward to compute for each element $e$ the elementary contributions $\mathbf{M}^{e}$ and $\mathbf{b}^{e}$. The latter is assembled to form the global "rigidity" matrix $\mathbf{M}$ and "force" vector $\mathbf{b}$, as in standard finite element problems. The only difference is that the "rigidity" matrix and the "force" vector contain picture gradients in addition to the shape functions, and the "force" vector includes also picture differences. The matrix $\mathbf{M}$ is symmetric, positive and sparse. These properties are exploited to solve the linear system efficiently. Last, the domain integrals involved in the expression of $\mathbf{M}^{e}$ and $\mathbf{b}^{e}$ require imperatively a pixel summation. The classical quadrature formulas (e.g., Gauss points) is not used because of the very irregular nature of the image texture. This last property is crucial to obtain an accurate displacement evaluation. Furthermore, sub-pixel interpolation is used to increase the resolution of the algorithm. The gradient of the image functions is obtained by performing Fourier transforms. The very use of a Taylor expansion requires that the displacement be small when compared with the correlation length of the texture. For a fine texture and large initial displacements, this requirement appears as inappropriate for convergence to a meaningful solution. Thus one may devise a generalization to arbitrarily expand the correlation length of the texture. This is achieved 
through a coarse-graining step. The interested reader may refer to Reference [3] for additional details concerning the above mentioned features.

\subsection{Extended algorithm: $X$-Q4-DIC}

As mentioned in the Introduction Section, the above-described discretization scheme is not suited to potentially discontinuous displacement fields. Furthermore, the real discontinuity of the displacement field (as what is observed from one face of a crack to the other one) is smoothed by the Q4P1 shape functions. This may bias the post-processing of the measured displacement to determine stress intensity factors $[18,13]$. It is proposed here to circumvent this difficulty in the spirit of X-FEM. The partition of unity property of finite element shape functions over the patch covered by the set of nodes $\mathcal{N}$

$$
\sum_{n \in \mathcal{N}} N_{n}(\mathbf{x})=1
$$

is exploited to enrich the kinematic discretization basis. The enriched discretization reads

$$
\mathbf{u}(\mathbf{x})=\sum_{n \in \mathcal{N}} \sum_{\alpha} a_{\alpha n} N_{n}(\mathbf{x}) \mathbf{e}_{\alpha}+\sum_{n \in \mathcal{N}_{e n r}} \sum_{\alpha} d_{\alpha n} N_{n}(\mathbf{x}) \varphi(\mathbf{x}) \mathbf{e}_{\alpha}
$$

where $\varphi$ is the enrichment function and $\mathcal{N}_{\text {enr }}$ is a sub-set of $\mathcal{N}$ wherein nodes hold enriched degrees of freedom $d_{\alpha n}$. The enrichment function is chosen arbitrarily to capture a specific singularity of the solution to the minimization problem. Let us now focus on a displacement field in the vicinity of a crack tip. The two components of the displacement have a discontinuity across the crack faces and an asymptotic behavior with super- and sub-singular terms around the crack tip [14]. In the original X-FEM, five enriched functions are used, namely, one which is discontinuous across the crack faces, and four singular functions. The approximation of the 
displacement is

$\mathbf{u}(\mathbf{x})=\sum_{n \in \mathcal{N}} \sum_{\alpha} a_{\alpha n} N_{n}(\mathbf{x}) \mathbf{e}_{\alpha}+\sum_{n \in \mathcal{N}_{\text {cut }}} \sum_{\alpha} d_{\alpha n} N_{n}(\mathbf{x}) H(\mathbf{x}) \mathbf{e}_{\alpha}+\sum_{n \in \mathcal{N}_{\text {tip }}} \sum_{\alpha} \sum_{j=1}^{4} c_{\alpha n j} N_{n}(\mathbf{x}) C_{j}(\mathbf{x}) \mathbf{e}_{\alpha}$

Four functions (only one is discontinuous across the crack faces) are used

$$
\left[C_{j}\right]=\left[\sqrt{r} \sin \left(\frac{\theta}{2}\right), \sqrt{r} \cos \left(\frac{\theta}{2}\right), \sqrt{r} \sin \left(\frac{\theta}{2}\right) \sin (\theta), \sqrt{r} \cos \left(\frac{\theta}{2}\right) \sin (\theta)\right]
$$

Enriched degrees of freedom associated with these functions are added to the sub-set of nodes $\mathcal{N}_{\text {tip }}$ whose support contains the crack tip (Figure 3). The support of a node is that of its finite element shape function i.e., the set of elements having the considered node within their connectivity. Concerning the discontinuous function, the symmetrized Heaviside function was originally used in X-FEM but the standard Heaviside function $\mathcal{H}$ can also be used $[24,23,25,17,5]$. We choose the alternative function proposed by $\mathrm{Zi}$ and Belytschko [26]. Each node whose support is completely cut by the crack surface (Figure 3) holds an additional degree of freedom for each component of the displacement associated with the function $H_{e}$

$$
H_{e}(\mathbf{x})=\mathcal{H}(\mathbf{x})-\mathcal{H}\left(\mathbf{x}_{e}\right)
$$

In this definition, the vector $\mathbf{x}_{e}$ gives the location of node $e$. Consequently, the enrichment function $H_{e}$ equals 0 over the area of the support of $e$ (i.e., elements that have $e$ within their connectivity) on the same side of the crack as $e$. The major advantage of this function is that it avoids so-called blending elements (elements in which the enrichment is not supported by a partition of unity, i.e., elements some nodes of which are not enriched). Furthermore, enriched terms of the matrix are only to be computed for the elements cut by the crack.

As mentioned in Section 2.2, Gauss quadrature is not used because of the image texture. The numerical integration is performed pixel-wise and any subdividing or special quadrature for 
the enriched elements are not needed. However, when a discontinuous kinematics is allowed for an element, the elementary matrix is to be computed over two different areas of the deformed image. Moreover, the extended interpolation scheme is introduced at the finest scale of the multi-scale algorithm. At the larger scale, the Q4 interpolation is assumed herein to be rich enough to capture the low spatial frequency content of the solution. Since the discontinuity is a very short wavelength phenomenon, the enriched functions are used only at the true scale (i.e., the finest one).

In order to evaluate the influence of the singular enrichment $(\sqrt{r}$ functions $)$, a strategy with only discontinuous enrichment is also tested. If the singular enrichment is not considered, the element containing the crack tip does not hold any discontinuity because the support of its nodes is not completely cut by the crack. Hence, as an alternative to what is described above, a strategy including only discontinuous enrichments for the nodes whose support is at least partially cut by the crack (in contrast to completely cut for the first strategy) is implemented. Consequently, in the element containing the tip, the nodes located behind the tip hold a discontinuous enrichment. Hence, the displacement field is discontinuous inside the tip element but the discontinuity vanishes on its edge in front of the tip. Figure 3 describes the two enrichment strategies.

\section{UNCERTAINTY ANALYSIS}

In this section, an uncertainty analysis is carried out. This study aims at evaluating the performance of the eXtended Digital Image Correlation (X-DIC) technique. As a preliminary test, it has been checked that the enrichment does not perturb the estimation of a uniform rigid body translation. Similar values for the displacement uncertainty, e.g., of the order of $10^{-3}$ pixel 
for a 32-pixel element size, are obtained with or without enrichment. The two enrichment strategies enable the DIC algorithm to capture a priori different types of displacement fields:

\subsection{Discontinuous rigid body translation}

First, a piecewise discontinuous rigid body translation is artificially imposed to a reference image. The image is $512 \times 512$ pixels with an 8-bit digitization. An artificial discontinuity is considered in the middle of the image, see Figure 1. Only half of the image is artificially translated in the range $[0,1]$ pixel by 0.1 -pixel increments in the horizontal as well as in the vertical direction. The region of interest for the correlation extends over the entire image. A patch of a number of elements depending upon their size is centered on the image. Consequently, the elements are not positioned so that the discontinuity cut them into two equal parts i.e., the relative position between the discontinuity and the elements varies with the element size. For each value of the artificial displacement and for each element size between 8 and 64 pixels, the uncertainty $\sigma_{u}$ is estimated as

$$
\sigma_{u}=\left\langle\left\|\mathbf{u}-\mathbf{u}_{p}\right\|^{2}\right\rangle^{1 / 2}
$$

where $\mathbf{u}_{p}$ is the prescribed displacement field, and $\langle\ldots\rangle$ refers to spatial averaging. Then, $\sigma_{u}$ is plotted as a function of the element size as an averaged value of the uncertainty obtained for the values of the prescribed displacements.

Figure 4 shows the results obtained with Q4 elements, and Q4 elements enriched with the discontinuous function $H_{e}$. By using a standard interpolation scheme, a divergence of the displacement uncertainty with the element size is observed. As the elements become larger, the solution is smeared out over a larger zone that induces larger uncertainties for the displacement estimate. If discontinuous functions are added to the interpolation, a decreasing 
trend is observed with an uncertainty as low as $2 \times 10^{-3}$ pixel for 64 -pixel elements. Figure 5 shows the uncertainty obtained on the displacement jump along the discontinuity itself. The uncertainty decreases as the element size increases. The performance of the proposed approach is illustrated here with an uncertainty level as low as $10^{-3}$ pixel for 64 -pixel elements.

\subsection{Crack displacement field}

Second, an elastic mixed mode crack displacement field is artificially prescribed to the reference image. The artificial crack is vertical, it starts in the middle of the bottom side of the image and the crack tip is located at the center. The same procedure as above is used and the center of the element containing the crack tip is not enforced to coincide with it. The amplitude of the artificial displacement is chosen so that, at the left edge of the image, mode I and II displacement jumps have the same value in the $[0,1]$-pixel range. This case is considered as particularly difficult since all the involved displacements are sub-pixel.

Figure 6 enables one to compare the results obtained by DIC with standard Q4 elements, with those obtained with a discontinuous enrichment and those obtained with discontinuous and singular enrichments. As expected from Figure 2, a poor performance is observed with a Q4 interpolation, whereas any enrichment strategy restores uncertainties of the order of $10^{-2}$ pixel. In this case, the $\sqrt{r}$-dominant zone extends over the entire image, the singular enrichment does not improve the displacement uncertainty compared to the strategy with discontinuous enrichment only. When the element size increases, the linear interpolation of the non-enriched elements is not able to capture the $\sqrt{r}$ behavior of the prescribed displacement. Consequently, the uncertainty increases.

The crack opening displacements obtained using the two enrichment strategies are plotted 
in Figure 8 for 16-pixel elements and in Figure 9 for 32-pixel elements. In the elements where the discontinuous enrichment is used in both strategies the results are very close. In the element containing the crack tip, the singular functions yield an exact kinematic location of the tip i.e., the crack opening displacement vanishes at the tip. On the contrary, when only discontinuous functions are used, the tip position is on the edge of the element, which leads to a less accurate determination of the crack opening displacement in the element containing the tip (Figure 9). This difference decreases when the element size decreases. Furthermore, the level of uncertainty obtained on the crack opening displacement is shown in Figure 7. First, for the two enrichment strategies the uncertainty decreases. Lower values are obtained with the singular enrichment that captures the $\sqrt{r}$-behavior more accurately. Then, the same behavior as for the uncertainty on the entire displacement map is observed.

These two uncertainty analyses illustrate the gain that is achieved when using enriched kinematics as compared to the performance obtained with a Q4 algorithm. Looking for crack tip fields with a $\sqrt{r}$-dominance, an optimal element size seems to exist. The extended interpolation improves the solution only inside the element wherein it is active. Because the Q4 interpolation remains valid over the rest of the elements, using larger elements means a decreasing uncertainty for the enriched element but a poorer performance over the rest of the mesh. As a limit case when the entire image is covered by one element, a fully integrated approach is probably more favorable [18]. However, the present technique is more flexible and applies to a larger number of applications, for example with two or more cracks. Furthermore, if the strategy with only a discontinuous enrichment is used, the limitations of Linear Elastic Fracture Mechanics may be investigated experimentally and numerically through applications where the elasto-plastic region extends over a large zone. In this case, the crack opening Copyright (C) 2000 John Wiley \& Sons, Ltd. Int. J. Numer. Meth. Engng 2000; 00:1-6 Prepared using nmeauth.cls 
is piecewise linear and the displacement interpolation wherein the elements surrounding the crack tip remains standard, namely no specific behavior in $r^{1 / n}$ is prescribed, where $n$ is the hardening exponent.

\subsection{Application to a Silicon Carbide sandwiched beam}

The performances of the proposed approach are now evaluated on an experimental case. The application discussed herein is based upon a Silicon Carbide sandwiched beam test (Figure 10a). This type of experimental set-up allows one to initiate a crack without complete failure of the central beam. However, the arrest conditions are strongly dependent upon the friction between the beams, their flexural stiffness, and the notch geometry [7]. Therefore, numerical tools may prove useless to evaluate stress intensity factors.

The region of interest covers the entire image whose size is $1008 \times 1016$ pixels (8-bit digitization). The mesh and the crack geometry are shown in Figure 10-b. Figure 11 shows the displacement fields obtained with the three interpolation schemes. First, Q4 elements, then enriched Q4 interpolation using the two different strategies. The first analysis using Q4 elements allows one to locate the crack path. Then, the enrichments are positioned on the mesh that consists of 32-pixel elements. The displacement discontinuity is better described with the enriched elements even if the error maps $\Phi$ remain similar (Figure 12). In this case, the maximum displacement jump is less than 0.5 pixel and the error that perturbs the standard Q4 estimate of the displacement reduces to the crack path itself. The average value of the residual error is around 2.95 gray level for the three discretization schemes but the maximum value of the residual error, which is 60 gray level for the Q4 interpolation decreases to 57 if any of the enrichment strategies is used. However, X-DIC enables one to measure a displacement 
field with an explicit discontinuity and an asymptotic $\sqrt{r}$-dependence.

The use of extended digital correlation enables one to measure discontinuous as well as asymptotic crack displacement fields. The preliminary uncertainty analysis on artificially deformed pictures illustrates the benefit of the proposed approach. By means of the enriched interpolation, it is possible to use, for the same level of accuracy, larger elements without increasing the measurement uncertainty. Furthermore, the analysis of an experimental case is presented, where the shape of the crack surface, which can be observed on the error map, is straight. This is however not a general result as demonstrated by Figure 17-c where a description of the crack faces by a straight line is not accurate. The next section aims at relaxing this hypothesis.

\section{CRACK SHAPE OPTIMIZATION}

As illustrated by Figure 17, the crack shape in samples may not be perfectly straight and a piecewise straight description of the crack geometry is inaccurate. Furthermore, as the above introduced enrichment functions depend upon the crack geometry, it must be described in an appropriate way. For instance, $H_{e}$ calls for the "above-bellow" information and the singular functions rely on the distance to the crack tip and angle with respect to a local crack tip co-ordinate system.

Level sets are used in a number of cases in combination with X-FEM [20, 21, 9]. This technique offers several advantages. Among them, an implicit description of the crack topology by contours of a level set function and a local curved co-ordinate system directly derived from the gradient of the level set. Consequently, enrichment functions are easily evaluated as level set functions. Moreover, efficient numerical techniques for moving level sets have been 
developed [19]. Then, the quantification of the gap between an initial, say straight, crack path and the real one, through moving level sets, will allow for the optimization of the crack geometry.

\subsection{Crack description by level sets}

Parameterizing the crack geometry requires the description of the crack surface, a crack path in $2 \mathrm{D}$, as well as the positioning of the crack tip on this surface, respectively tip on this path. Hence, the crack surface is implicitly defined as the 0 -contour of a first level set $\psi_{c}$. Then the crack tip is defined as the intersection between the crack surface $\left(\psi_{c}=0\right)$ and the 0 -contour of a front level $\psi_{f}$ (see Figure 13):

$$
\psi_{c}=0 \text { on the crack, }\left(\psi_{c}=0, \psi_{f}=0\right) \text { is the crack tip }
$$

The signed distance is used as level set. Therefore, the norm of the gradient of both level sets is 1

$$
\left|\nabla \psi_{c}\right|=1 \quad\left|\nabla \psi_{f}\right|=1
$$

Furthermore, if $\nabla \psi_{f}$ is orthogonal to $\nabla \psi_{c}$

$$
\nabla \psi_{c} . \nabla \psi_{f}=0
$$

then $\left(\psi_{f}, \psi_{c}\right)$ are used as a local co-ordinate system. The enrichment functions are now evaluated with two level sets. The Heaviside function $\mathcal{H}$ is

$$
\mathcal{H}(\mathbf{x})=\frac{1}{2}\left(\operatorname{sign}\left(\psi_{c}\right)+1\right)
$$

For the singular function, the distance to the crack tip and the angle $\theta$ are obtained from the following formulas

$$
r=\sqrt{\psi_{c}^{2}+\psi_{f}^{2}} \quad \theta=\tan ^{-1}\left(\frac{\psi_{c}}{\psi_{f}}\right)
$$




\subsection{Level set propagation}

Let $\psi$ be a level set. The total derivative of $\psi$ with respect to a virtual time $t$ at points following the initial 0 -contour is

$$
\frac{\partial \psi}{\partial t}+\mathbf{V} \cdot \nabla \psi=0
$$

where $\mathbf{V}$ is the velocity of the contour. Because the normal to the contour is given by the gradient of $\psi$, this equation is recast as

$$
\frac{\partial \psi}{\partial t}+F|\nabla \psi|=0
$$

where $F$ is the normal velocity of the considered contour. For numerical purposes, the time integration is performed by using an Euler scheme. The level set function is discretized in space by finite differences on the regular pixel grid of the image (not using the nodes of the finite element mesh). However, special care should be taken in order to deal with discontinuous derivatives. A Godunov scheme [8] is used to compute $F|\nabla \psi|$ in Equation (26), which is rewritten as

$$
\psi_{n+1}=\psi_{n}-\Delta t \nabla_{G}
$$

where $\Delta t$ is the time step whose critical value is $\Delta x / \max (|F|), \Delta x$ the grid step, and $\nabla_{G}$ the Godunov numerical elaboration of $F|\nabla \psi|$

$$
\nabla_{G}=\max (F, 0) \nabla^{+}+\min (F, 0) \nabla^{-}
$$


where $\nabla^{+}$and $\nabla^{-}$depend upon the finite difference gradient of $\psi, D^{x+}, D^{x-}$ and $D^{y+}, D^{y-}$

$$
\begin{gathered}
D^{x-}=\frac{\psi_{x}-\psi_{x-\Delta x}}{\Delta x} \quad D^{x+}=\frac{\psi_{x+\Delta x}-\psi_{x}}{\Delta x} \\
D^{y-}=\frac{\psi_{y}-\psi_{y-\Delta y}}{\Delta y} \quad D^{y+}=\frac{\psi_{y+\Delta y}-\psi_{y}}{\Delta y} \\
\nabla^{+}=\left[\max \left[P\left(D^{x-}\right)^{2}, N\left(D^{x+}\right)^{2}\right]+\max \left[P\left(D^{y-}\right)^{2}, N\left(D^{y+}\right)^{2}\right]\right]^{1 / 2} \\
\nabla^{-}=\left[\max \left[N\left(D^{x-}\right)^{2}, P\left(D^{x+}\right)^{2}\right]+\max \left[N\left(D^{y-}\right)^{2}, P\left(D^{y+}\right)^{2}\right]\right]^{1 / 2}
\end{gathered}
$$

where $P(z)$ and $N(z)$ denote respectively the positive and negative parts of a real number $z$.

After propagating the level set, a re-initialization step to the signed distance is performed. This is achieved by solving the following equation until steady state is reached

$$
\frac{\partial \psi}{\partial t}+\operatorname{sign}(\psi)(|\nabla \psi|-1)=0
$$

Numerically, the Godunov scheme is adapted in the following way

$$
\nabla_{\text {Ginit }}=\max (\operatorname{sign}(\psi), 0)\left(\nabla^{+}-1\right)+\min (\operatorname{sign}(\psi), 0)\left(\nabla^{-}-1\right)
$$

This re-initialization step does not change the 0-contour if the re-initialization velocity $(\operatorname{sign}(\psi))$ is equal to 0 on it. This is achieved by adopting an appropriate definition of the sign function, $\operatorname{sign}(0) \equiv 0$.

\subsection{Objective function for shape optimization}

Moving the crack surface, i.e., propagating the level set $\psi_{c}$, requires a normal velocity $F$. A mispositioning of the crack surface induces higher values of the error $\Phi$ as illustrated in Figure 14 . This error map will be used in order to propagate the level set, so as to resolve at best the crack meandering. To illustrate the proposed procedure, two test cases are first presented. In both cases, a discontinuous horizontal translation is artificially imposed to the part of the image that is located on the right of curved boundary. Two examples of the latter are considered, 
first a sinusoidal curve, and second a Gaussian one. The image is $512 \times 512$ pixels with an 8-bit digitization. The amplitude of the sinusoid is 5 pixels and its period is 128 pixels. The prescribed displacement is 0.5 pixel. The Gaussian curve has a 10 pixel amplitude, and extends over 60 pixels. Figure 14 shows the error map obtained with a discontinuous enrichment along a straight line. The area surrounded by the real discontinuity line and the straight one exhibits higher error levels. The use of $\Phi$ as an error indicator is straightforward in the co-ordinate system $\left(\psi_{f}, \psi_{c}\right)$. The initial discontinuity line is then to be moved by $\Delta$ so that

$$
\Delta\left(\psi_{f}\right)=\psi_{c}\left(\mathbf{x}_{\max }\left(\psi_{f}\right)\right)
$$

where $\mathbf{x}_{\max }\left(\psi_{f}\right)$ gives the location of the pixel on a $\psi_{f}$-contour where the error is maximum

$$
\Phi\left(\psi_{f}, \psi_{c}\left(\mathbf{x}_{\max }\right)\right)=\max _{\psi_{c}} \Phi\left(\psi_{f}, \psi_{c}\right)
$$

Then, the velocity $F$ is set to $\Delta$ and the propagation of the "crack" level set is performed over a virtual time of 1 . This procedure is included within the iterative determination of the displacement field. Since the local indicator $\Phi$ is based on the global residual, the solution now results from a minimization of $\Phi$ with respect to the displacement field and the crack geometry. Figure 15 illustrates the results obtained by using this procedure. The zone of higher error now reduces to the crack path itself and the optimized discontinuity is close to the real one. With shape optimization, the mean positioning error is 1.59 pixel for the sinusoidal discontinuity and 1.35 pixel for the Gaussian discontinuity. Furthermore, the positioning uncertainty is 1.43 pixel for the sinusoidal discontinuity and 0.93 pixel for the Gaussian discontinuity. The comparison between the displacement field measured using the optimized geometry and the one measured assuming a straight discontinuity is shown in Figure 16. With a straight discontinuity, the displacement cannot describe the real shape of the discontinuity 
and a mean error of 1.6 gray levels within the elements cut by the sinusoidal discontinuity is observed. By using the optimization of the crack shape, the error reduces to 1.1 gray levels and the displacement describes the imposed motion more accurately. The uncertainty in the displacement jump determination is 0.028 pixel for the sinusoidal discontinuity and 0.015 pixel for the Gaussian discontinuity. These results are to be compared with the uncertainty level for a known discontinuity (see Figure 5), which is about 0.003 pixel for the same element size (i.e., 32 pixels). This result shows that most of the uncertainties are related to a good positioning of the discontinuity profile. The same type of conclusion was reached when studying a cracked ceramic for which the uncertainties related to identified values of the stress intensity factor are mainly caused by the uncertainty associated with the positioning of the crack tip [18].

\subsection{Application to a CCT specimen}

The sample has a CCT geometry (Figure 17-a) and is subjected to cyclic tension with a load ratio $R=0.4$. In the present analysis, only the stage corresponding to the maximum load level is considered after about 300,000 cycles for which the crack size $2 a=14.5 \mathrm{~mm}$. Pictures are taken by using a long distance microscope and a CCD camera (resolution: $1024 \times 1280$ pixels, dynamic range: 12 bits) so that the physical size of one pixel is $2.08 \mu \mathrm{m}$. At this magnification, the raw surface is observed (Figure 17b). The studied material is an XC48 (or C45) steel [14].

The normal displacement map obtained with 32-pixel Q4 elements is shown in Figure 18a. Since the crack geometry is not straight, the Q4 interpolation does not give accurate results with this element size. Furthermore, the crack path is again smeared out by the continuous interpolation. Figure 18 also shows the displacement field obtained with a discontinuous interpolation and a straight crack (b) and the optimized crack (c). Furthermore, Figure 19 
shows the residual maps in gray levels. The first map is obtained with standard Q4 elements and a quite large error zone appears in the neighborhood of the crack. The value of the error is reduced by using discontinuous interpolation functions and a straight crack. The region of maximum error finally reduces to the crack path itself when the crack geometry optimization is used in combination with the extended interpolation. This is illustrated in Figure 20 where the initial and optimized crack fronts are superimposed onto the error maps. The improvement due to the crack geometry optimization is $3 \%$ in the elements cut by the crack when compared to the error obtained with the straight crack. When a standard interpolation is used, the average value of the residual error is 932 gray level whereas it decreases to 914 for a discontinuous enrichment associated with a straight crack and down to 911 for the optimized crack geometry. Note that the gray level scale has been limited to the range $[0 ; 5000]$ (hence saturating the largest values) in order to make the differences more readable. The actual dynamic range is 65536 gray levels. Figure 21 shows a comparison between the displacement jump obtained with a straight discontinuity and the optimized one. Fluctuations are observed with the optimized crack geometry when the crack shape is tortuous. However, the crack shape optimization enables for a better (i.e., less erratic) prediction of the displacement jump as the discontinuity introduced in the description of the kinematics better describes the actual one.

\section{CONCLUSION}

When using image correlation algorithms, the measurement uncertainty decreases when the element size increases. Therefore, it is difficult, if not impossible, to measure accurately displacement discontinuities when the element size increases. In this context, extended interpolation schemes, such as those proposed in X-FEM, are appealing since they allow 
for the description of discontinuities in the displacement field even when a coarse mesh is used. This paper presents an extended finite element formulation suitable for digital image correlation. Discontinuous as well as singular enrichment functions are introduced in the interpolation exploiting the partition of unity property of finite element shape functions. Artificial test cases as well as experimental studies show the advantages of the method for cracked specimens. In one of the experimental cases where the crack path was not straight, a crack shape optimization algorithm based on a two-scale multi-field minimization of the correlation residual was presented.

The proposed technique, which offers the ability of describing the displacement jump as well as the crack faces geometry, is more flexible than the "integrated approach" previously proposed to analyze cracked surfaces [18]. However, contrary to the latter approach, the stress intensity factors are not a direct output of the analysis and the values obtained by using leastsquares $[11,18,14]$ or (optimized) interaction integrals [16] may turn out to be sensitive to the enrichment used. This question constitutes the next step of the present work. Furthermore, the proposed approach unifies the interpolations used for experimental measurements and numerical simulations of cracked solids.

Last, there are other situations where the (strong) discontinuity kinematics introduced herein is significant. Among them, the analysis of friction between different parts in an assembly is made possible with a very good spatial resolution [15]. Another situation concerns weak discontinuities involved in localized phenomena (e.g., Piobert-Lüders [6] or Portevin-Le Châtelier bands [3]). The same type of procedure may be applied with a suitable enriched kinematics. 


\section{REFERENCES}

1. Babuska I, Melenk J. The Partition of unity method. International Journal for Numerical Methods in Engineering 1997; 40: 727-758.

2. Belytschko T, Moës N, Usui S, Parimi C. Arbitrary discontinuities in finite elements. International Journal for Numerical Methods in Engineering 2001; 50(4): 993-1013.

3. Besnard G, Hild F, Roux S. 'Finite-element' displacement fields analysis from digital images: Application to Portevin-Le Châtelier bands. Experimental Mechanics 2006; 46: 789-803.

4. Black T, Belytschko T. Elastic crack growth in finite elements with minimal remeshing. International Journal for Numerical Methods in Engineering 1999; 45: 601-620.

5. de Borst R, Remmers J, Needleman A. Mesh-independent numerical representations of cohesive-zone models. Engineering Fracture Mechanics 2006; 173(2): 160-177.

6. Fayolle X, Calloch S, Hild F. Controlling testing machines with digital image correlation. Experimental Techniques 2007; in press.

7. Forquin P, Rota L, Charles Y, Hild F. A method to determine the toughness scatter of brittle materials. International Journal of Fracture 2004; 125(1): 171-187.

8. Godunov S. A finite difference method for the numerical computation of discontinuous solutions of the equations of fluid dynamics. Math. Sb 1959; 47(3): 271-290.

9. Gravouil A, Moës N, Belytschko T. Non-planar 3D crack growth by the extended finite element and level sets. Part II: Level set update. International Journal for Numerical Methods in Engineering 2002; 53(11): 2569-2586.

10. Hild F, Raka B, Baudequin M, Roux S, Cantelaube F. Multi-scale displacement field measurements of compressed mineral wool samples by digital image correlation. Applied Optics 2002; ID 41(32): 6815-6828.

11. McNeill S, Peters W, Sutton M. Estimation of stress intensity factor by digital image correlation. Engineering Fracture Mechanics 1987; 28(1): 101-112.

12. Moës N, Dolbow J, Belytschko T. A finite element method for crack growth without remeshing. International Journal for Numerical Methods in Engineering 1999; 46(1): 133-150.

13. Réthoré J, Gravouil A, Morestin F, Combescure A. Estimation of mixed-mode stress intensity factors using digital image correlation and an interaction integral. International Journal of Fracture 2005; 132(1): 65-79.

14. Hamam R, Hild F, Roux S. Stress intensity factor gauging by digital image correlation: Application in cyclic fatigue. Strain 2006; accepted.

Copyright (c) 2000 John Wiley \& Sons, Ltd.

Int. J. Numer. Meth. Engng 2000; 00:1-6

Prepared using nmeauth.cls 
15. Réthoré J, Roux S, Hild F. From pictures to extended finite elements: Extended digital image correlation. Comptes Rendus de Mecanique 2007; in press.

16. Réthoré J, Roux S, Hild F. Noise-robust Stress Intensity Factor Determination from Kinematic Field Measurements. Engineering Fracture Mechanics 2007; accepted.

17. Remmers J, de Borst R, Needleman A. A cohesive segments method for the simulation of crack growth. Computational Mechanics 2003; 31: 69-77.

18. Roux S, Hild F. Stress intensity factor measurement from digital image correlation: post-processing and integrated approaches. International Journal of Fracture 2006; 140(1-4): 141-157.

19. Sethian J. Level set methods and fast marching methods: evolving interfaces in computational geometry, fluid mechanics, computer vision, and material science Cambridge University Press Cambridge 1996.

20. Stolarska M, Chopp D, Moës N, Belytschko T. Modeling crack growth by level sets in the extended finite element method. International Journal for Numerical Methods in Engineering 2001; 51: 943-960.

21. Sukumar N, Chopp D, Moran B. Extended finite element method and fast marching method for threedimensional fatique crack propagation. Engineering Fracture Mechanics 2000; 190: 6183-6200.

22. Sutton M, McNeill S, Helm J, Chao Y. Advances in two-dimensional and three-dimensional computer vision. In Photomechanics, Springer, 2000; 323-372.

23. Wells G, Sluys L. Discontinuous analysis of softening solids under impact loading. International Journal for Numerical and Analytical Methods in Geomechanics 2001; 25: 691-709.

24. Wells G, Sluys L. A new method for modeling cohesive cracks using finite elements. International Journal for Numerical Methods in Engineering 2001; 50: 2667-2682.

25. Wells G, Sluys L, de Borst R. Simulating the propagation of displacement discontinuities in a regularized strain-softening medium. International Journal for Numerical Methods in Engineering 2002; 53: 1235-1256.

26. Zi G, Belytschko T. New crack-tip elements for XFEM and applications to cohesive cracks. International Journal for Numerical Methods in Engineering 2003; 57(15): 2221-2240.

Copyright (C) 2000 John Wiley \& Sons, Ltd.

Int. J. Numer. Meth. Engng 2000; 00:1-6

Prepared using nmeauth.cls 


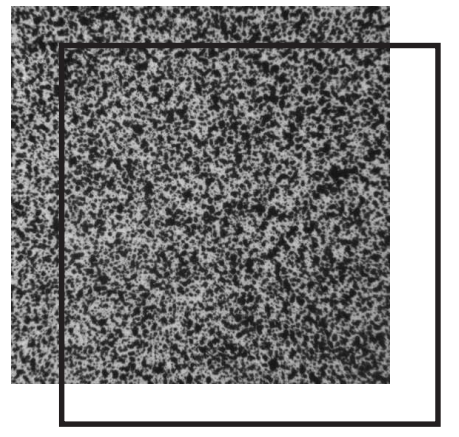

$-a-$

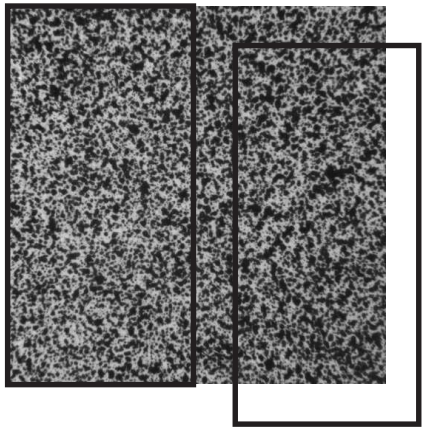

$-b-$

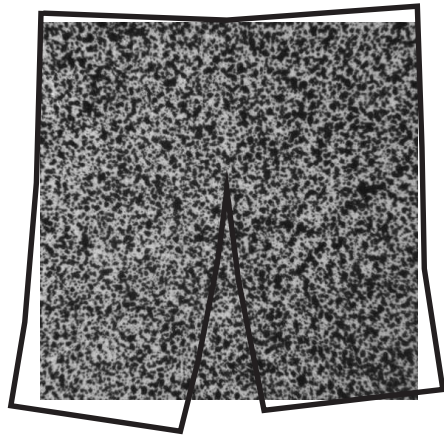

$-\mathrm{C}-$

Figure 1. Various artificial motions applied to a test image. -a-Rigid body translation. -b-Piecewise rigid body translation. -c-Mixed mode asymptotic crack tip field. 


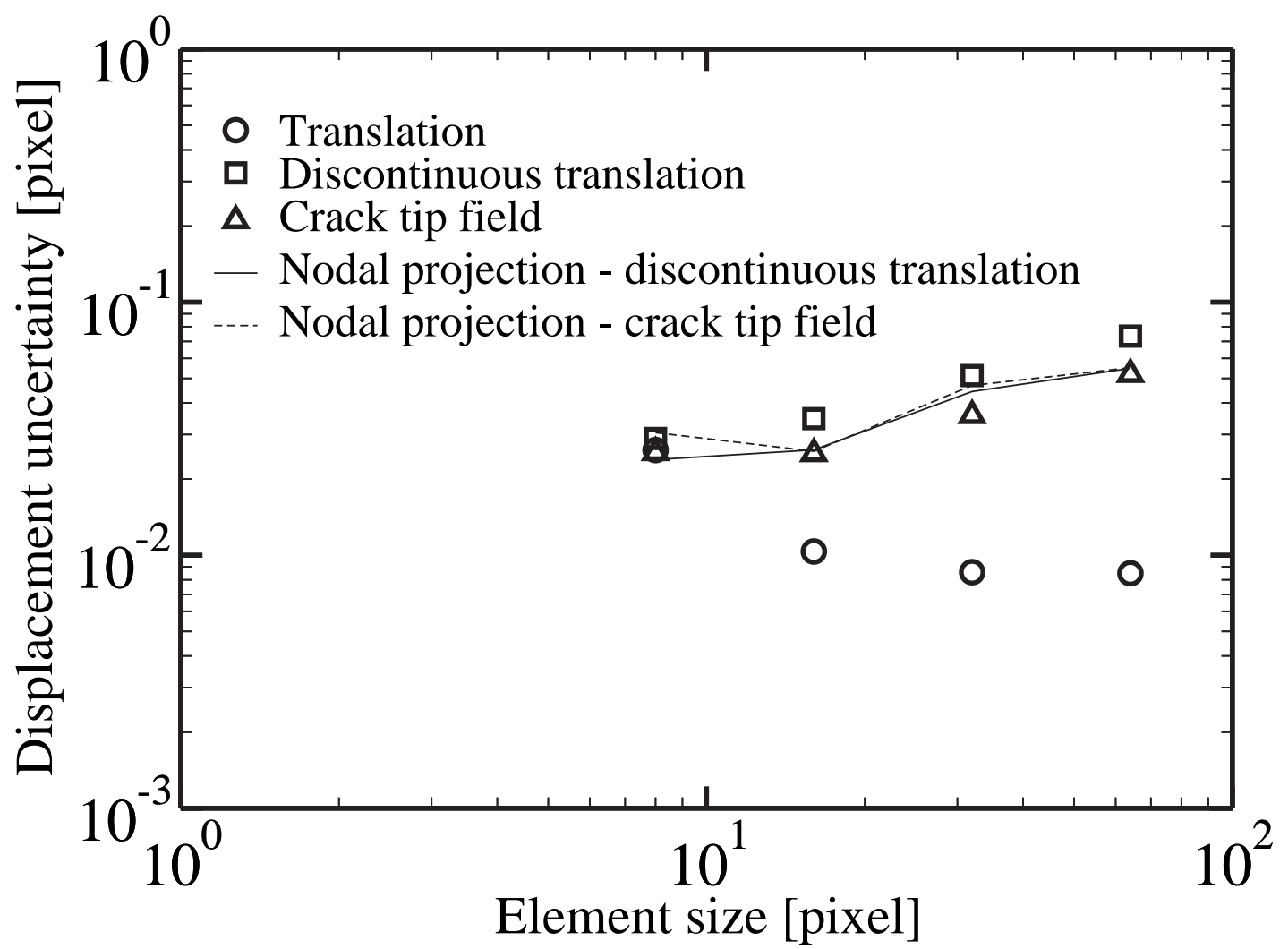

Figure 2. Displacement uncertainty for a Q4-algorithm when a rigid body translation, a piecewise translation or an asymptotic crack tip field are artificially prescribed to the reference picture of Figure 1 (resolution: $512 \times 512$ pixels, dynamic range: 8 bits). 


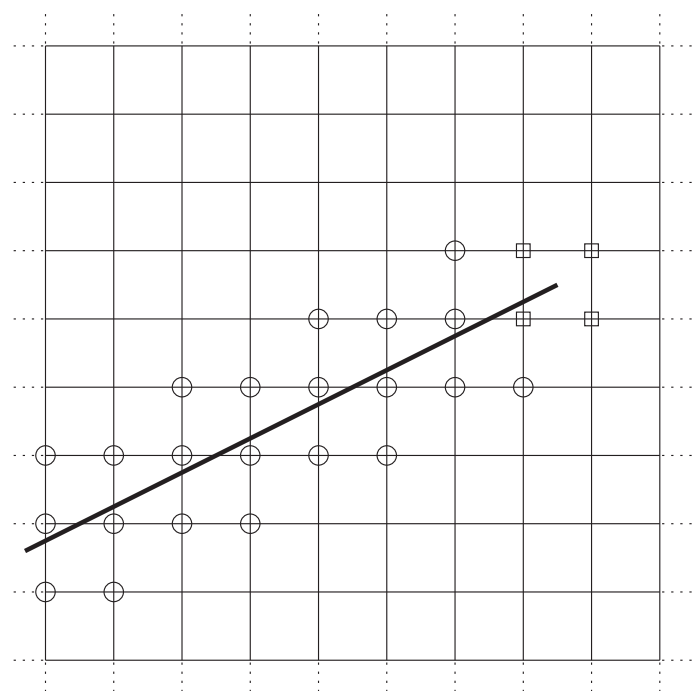

Discontinuous + singular enrichments

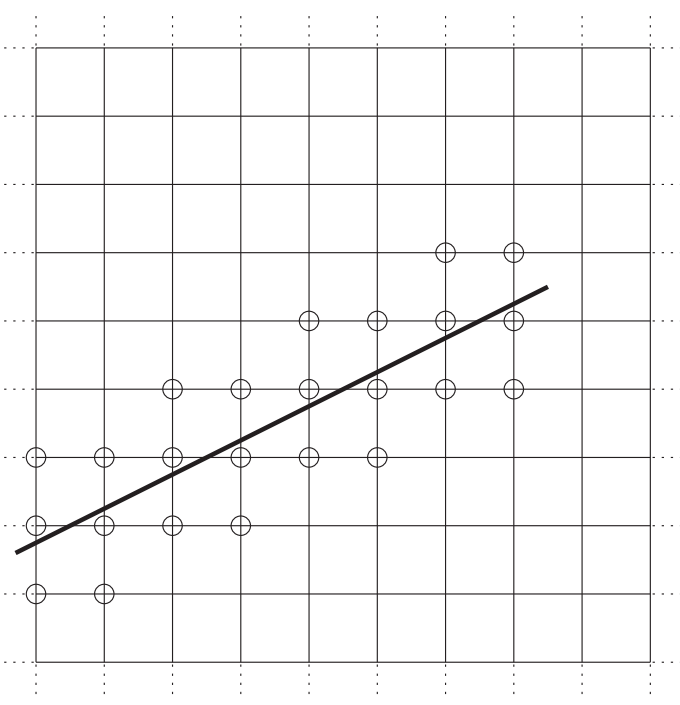

Discontinuous enrichments

Figure 3. Typical enrichment configuration: circles denote nodes with discontinuous additional degrees of freedom and squares nodes with singular additional degrees of freedom. 


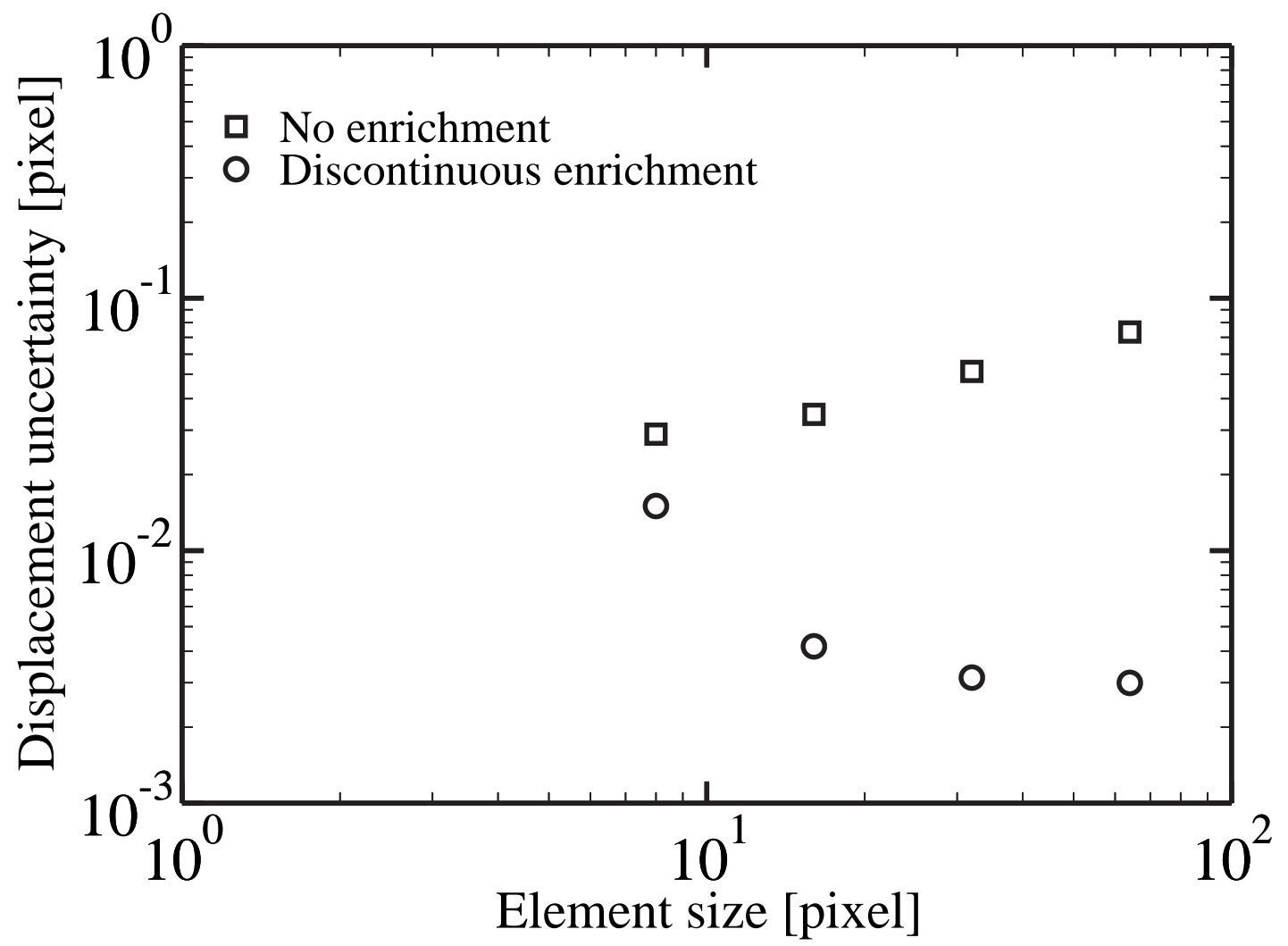

Figure 4. Displacement uncertainty for a discontinuous translation obtained with standard Q4 finite elements and Q4 elements enriched with a discontinuous function. 


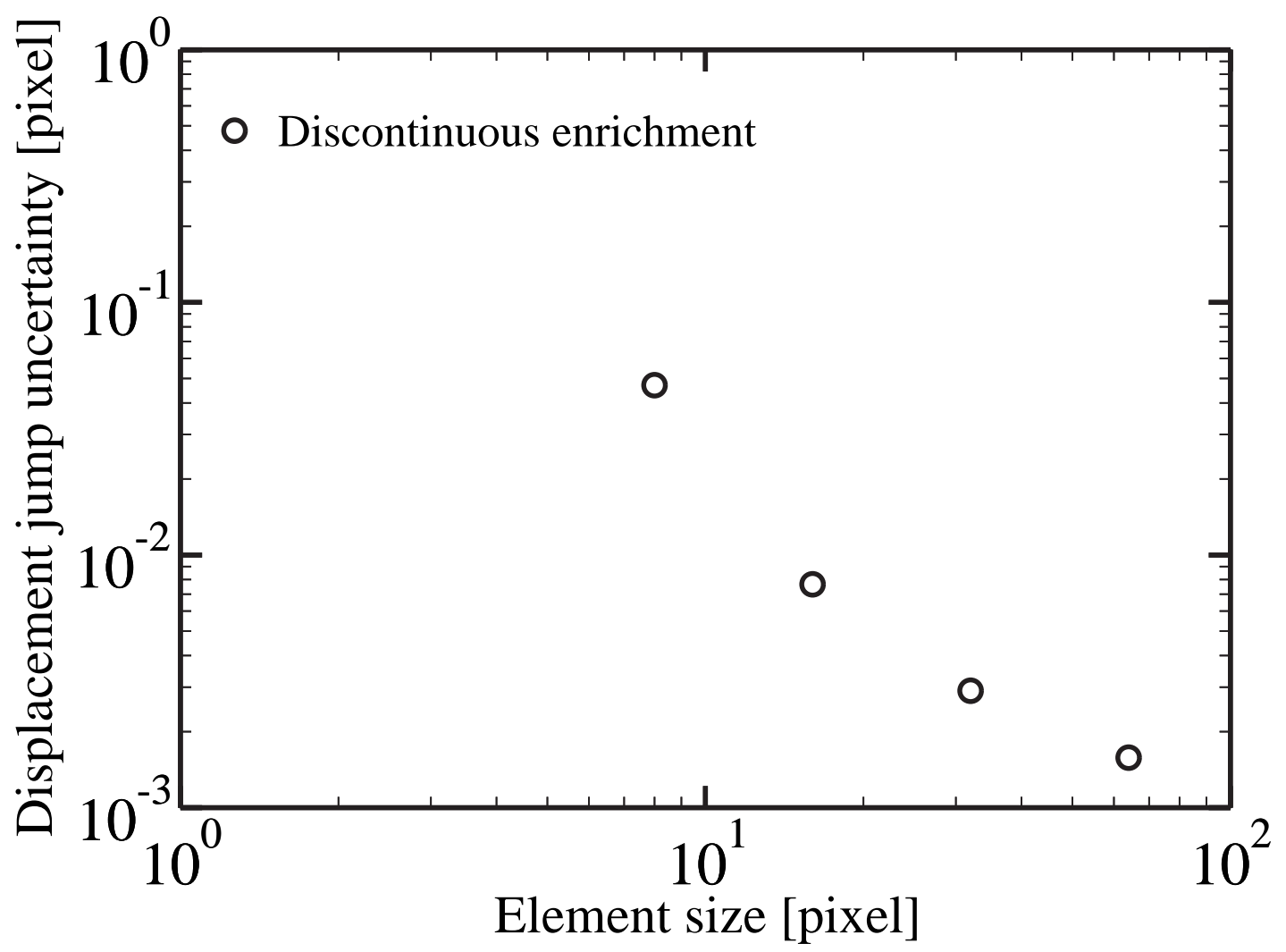

Figure 5. Displacement jump uncertainty for a discontinuous translation obtained with Q4 elements enriched with a discontinuous function. 


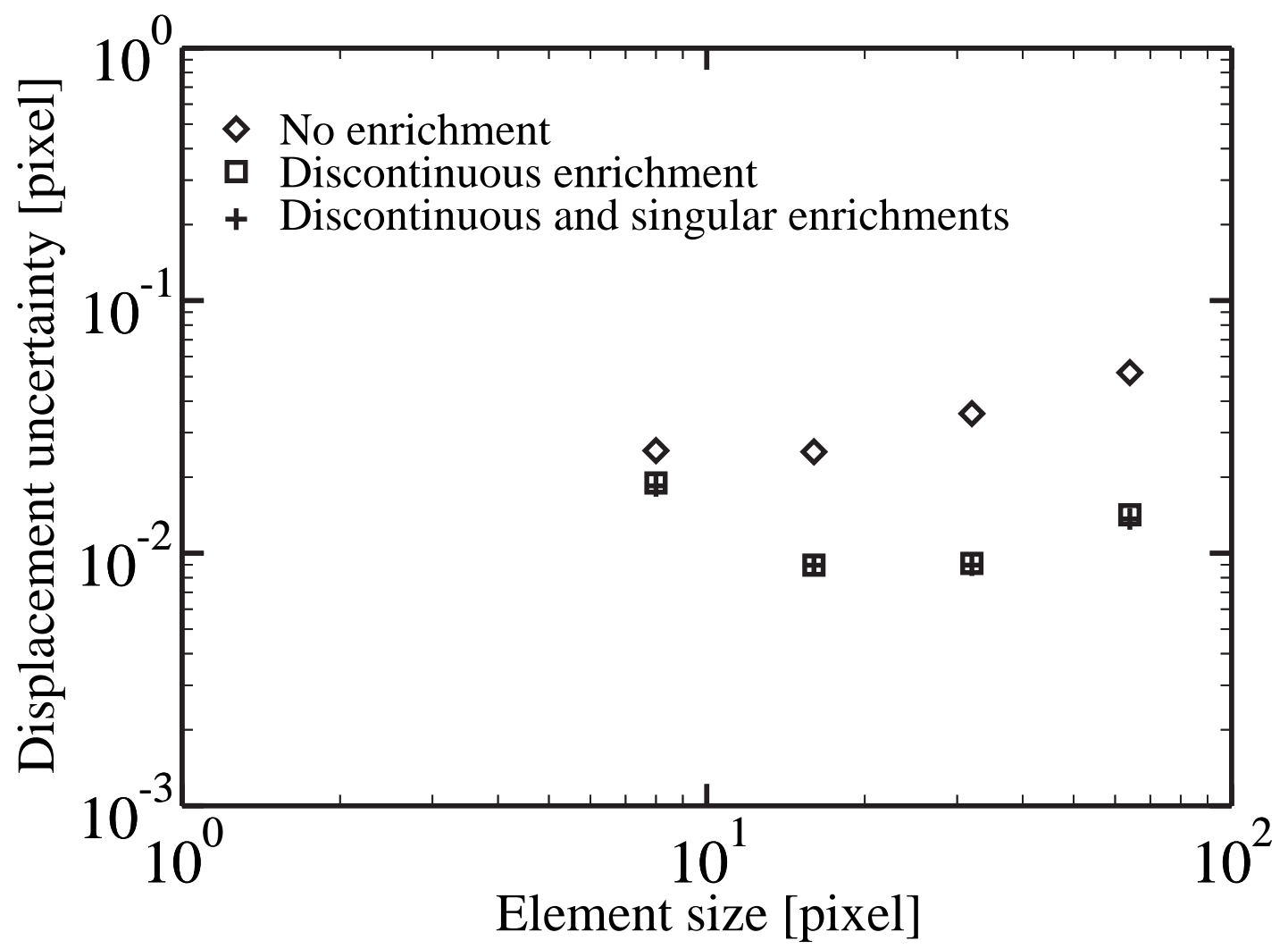

Figure 6. Displacement uncertainty for a crack tip field obtained with Q4 finite elements, Q4 elements enriched with a discontinuous function and Q4 elements enriched with a discontinuous function and tip functions. 


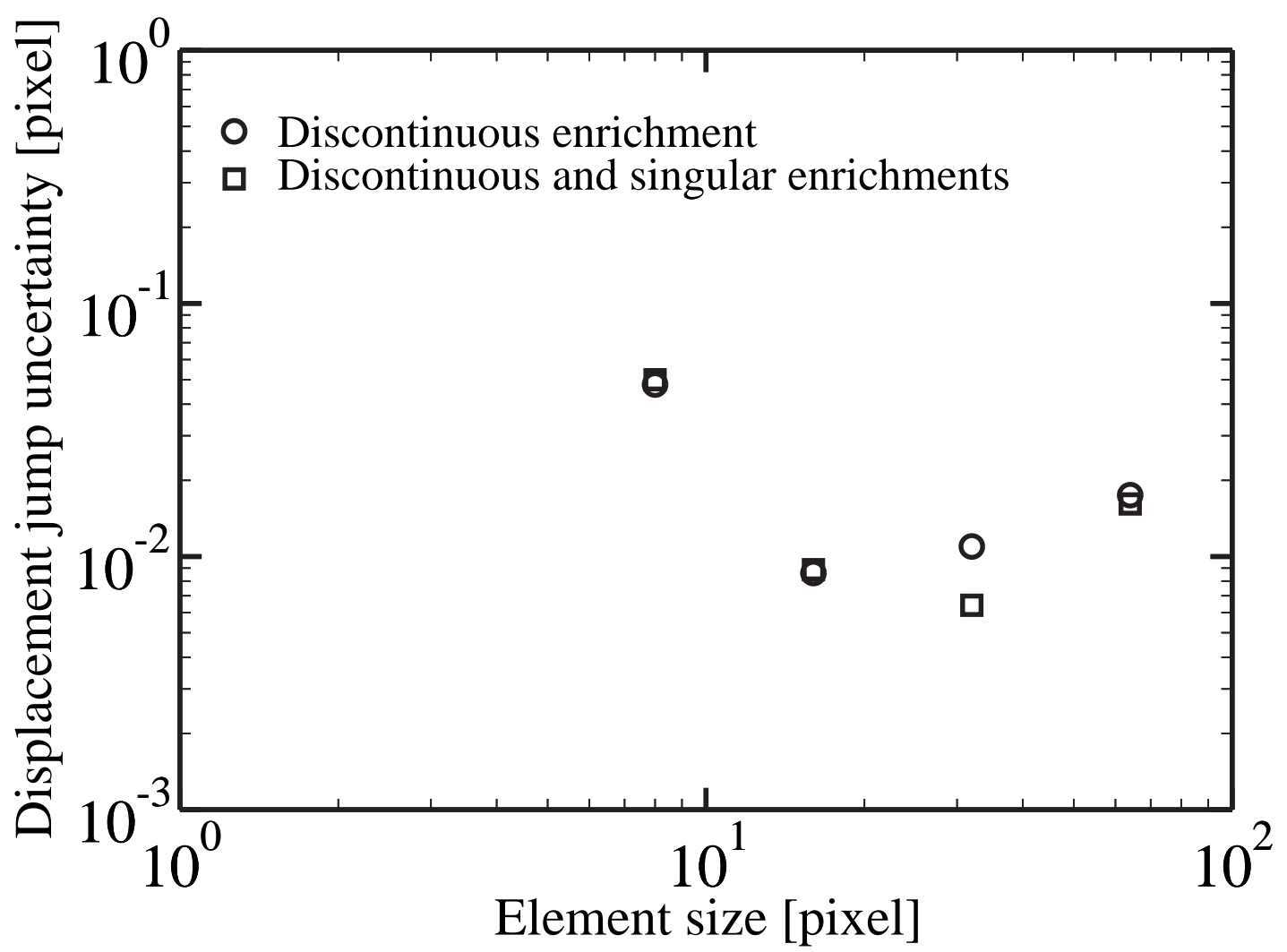

Figure 7. Displacement jump uncertainty for a crack tip field obtained Q4 elements enriched with a discontinuous function and Q4 elements enriched with a discontinuous function and tip functions. 


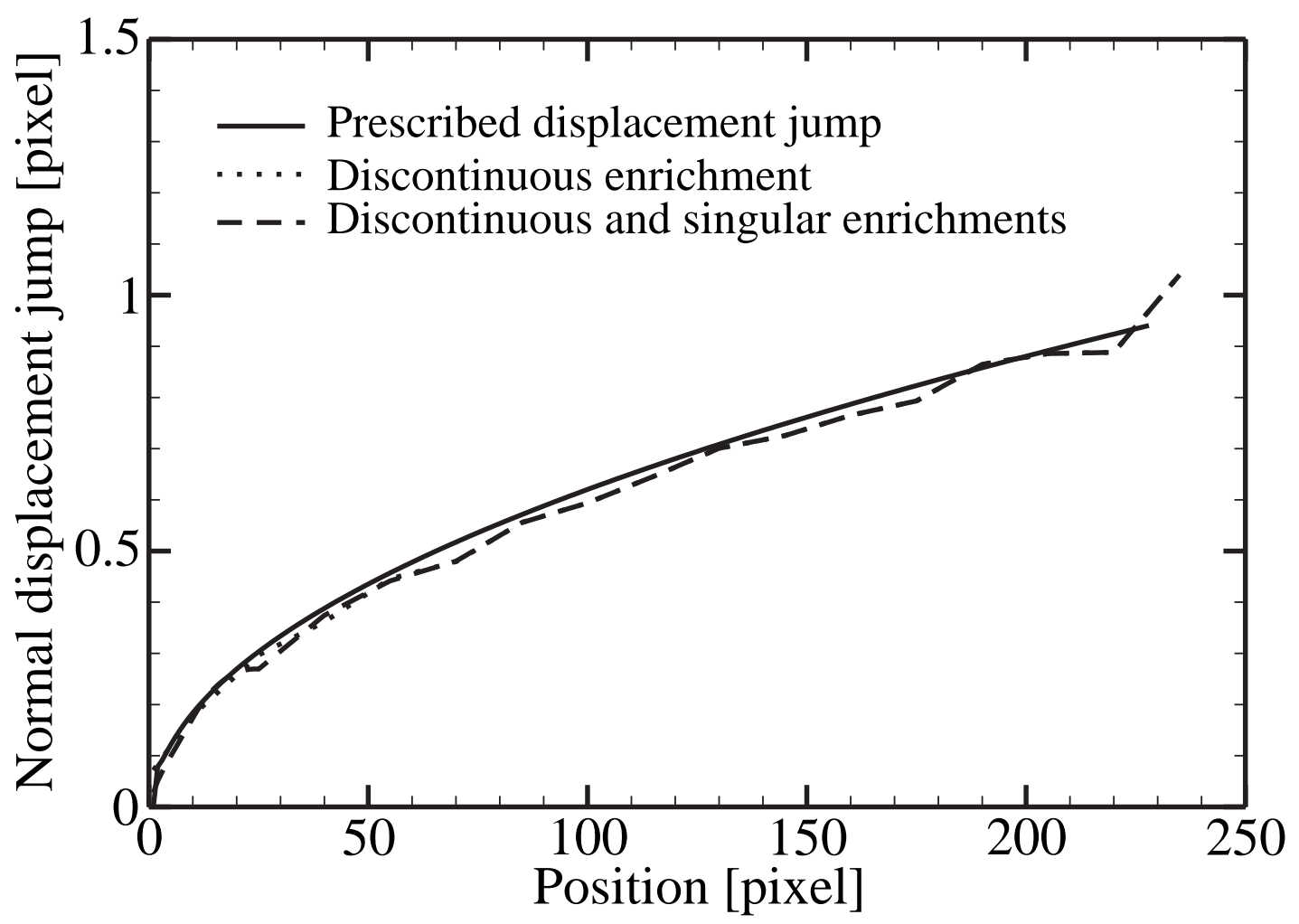

Figure 8. Displacement jump for 16-pixel elements using a discontinuous function and using discontinuous and tip functions. On the boundary of the image the enriched degrees of freedom are less constrained then in the interior, then boundary effects are observed. The larger the elements the more constrained are the enriched degrees of freedom and the less are the boundary effects 


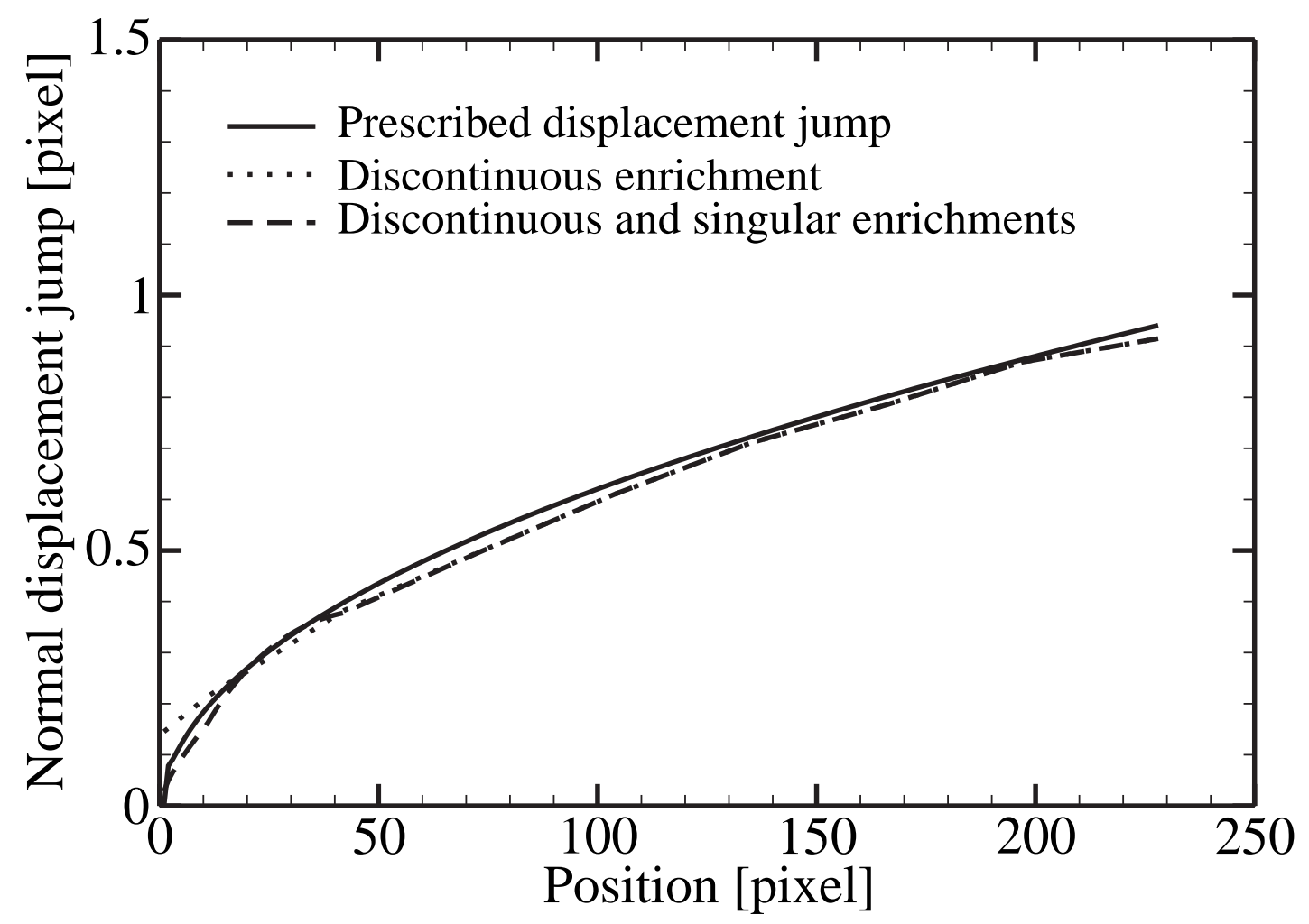

Figure 9. Displacement jump for 32-pixel elements using a discontinuous function and using discontinuous and tip functions. Here, because of a larger element size the boundary effect is less important. 


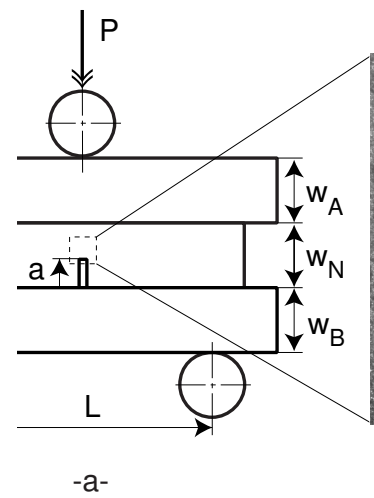

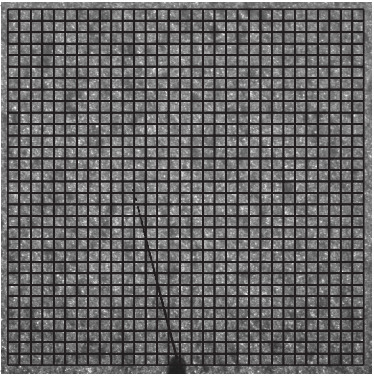

$-b-$

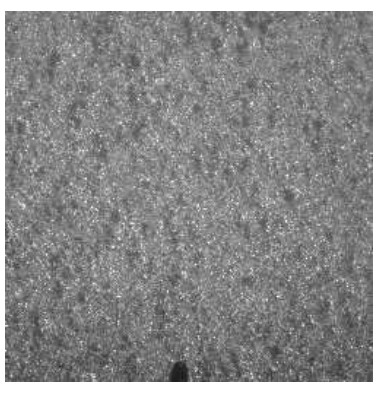

$-c-$

Figure 10. Geometry of the sandwiched beam set-up (a). The brittle sample (N) is put in between two steel beams (A and B). A three-point bend test is performed. A first stable crack is initiated. Initial (b) and cracked (c) SiC sample face with a 32-pixel elements mesh and the assumed crack geometry. In the middle of the lower side, the black spot is the mark of the notch, from which a crack propagates. The image size is $1008 \times 1016$ pixels with a conversion factor $p=1.85 \mu \mathrm{m} /$ pixel. 

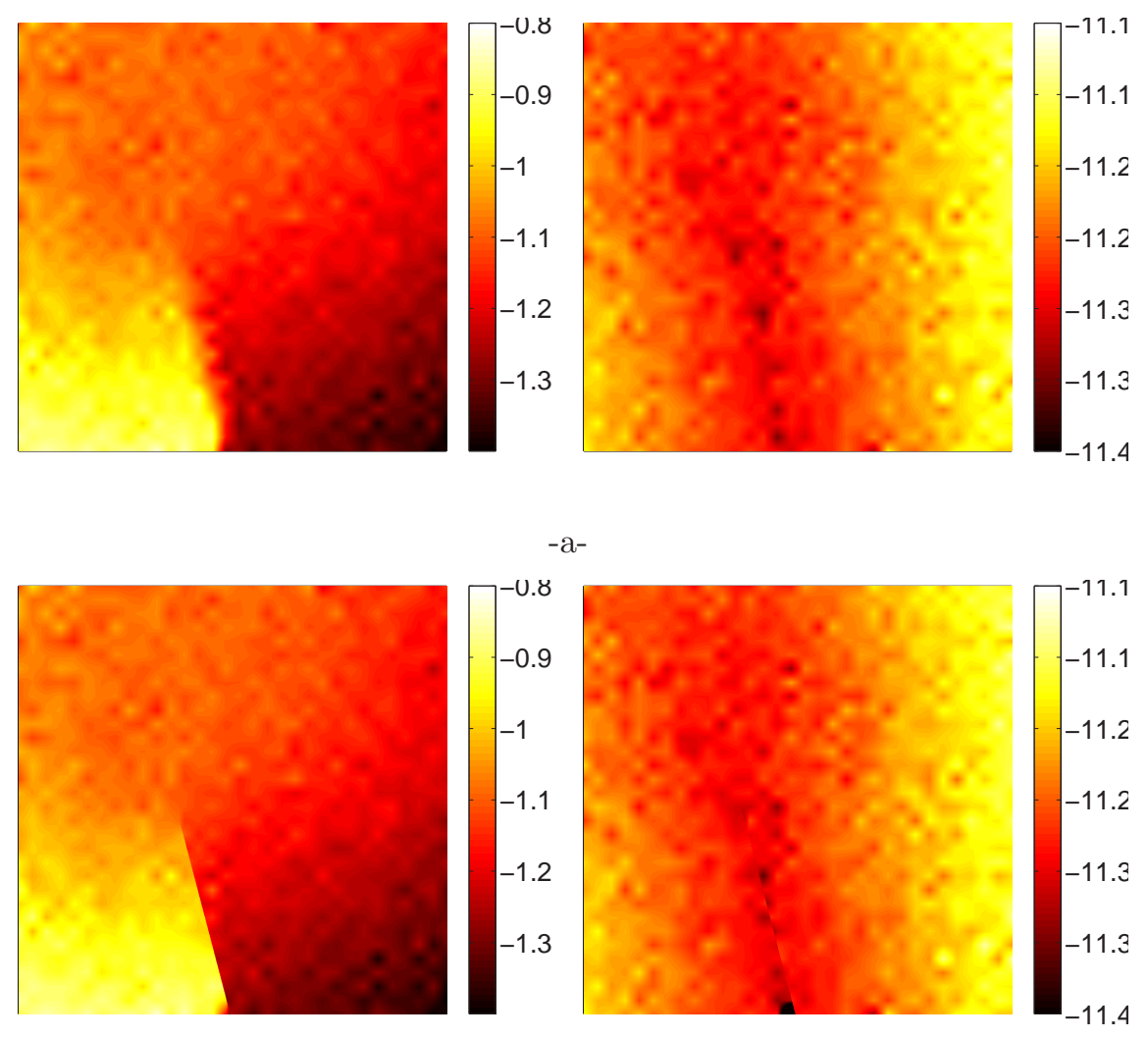

$-b-$
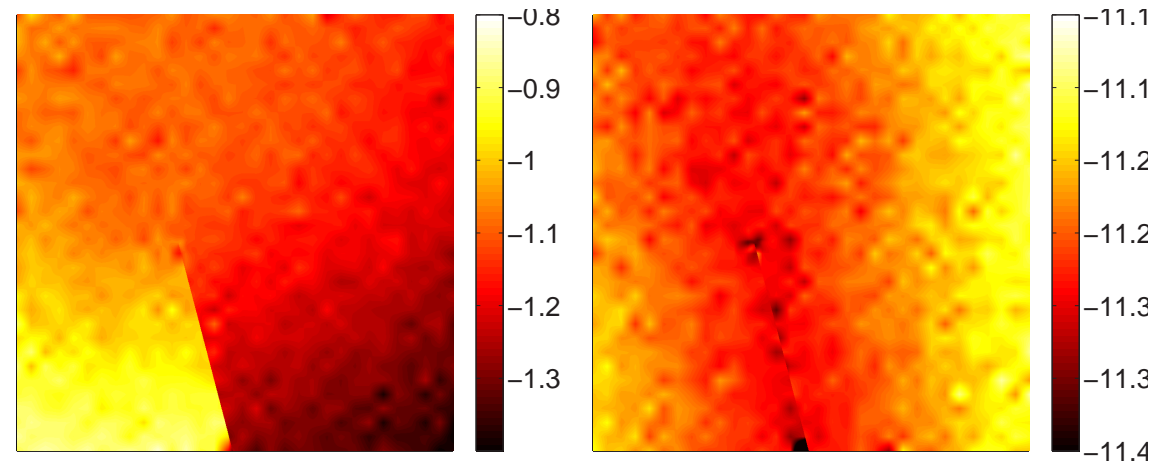

$-\mathrm{c}-$

Figure 11. Normal (left) and tangential (right) displacement map for the $\mathrm{SiC}$ specimen for 32pixel elements using: no enrichment (a), discontinuous enrichment (b), discontinuous and singular enrichments (c). The conversion factor is $p=1.85 \mu \mathrm{m} /$ pixel. 


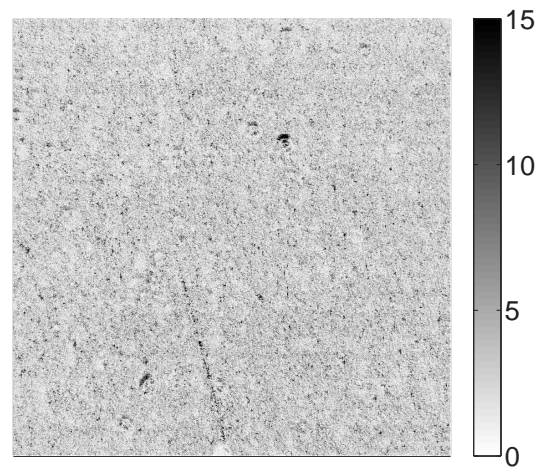

$-a-$

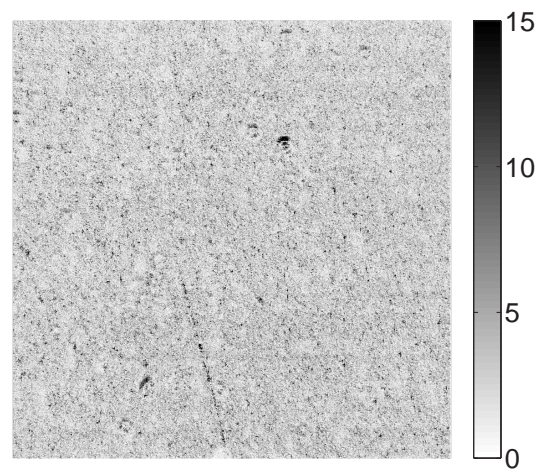

$-b-$

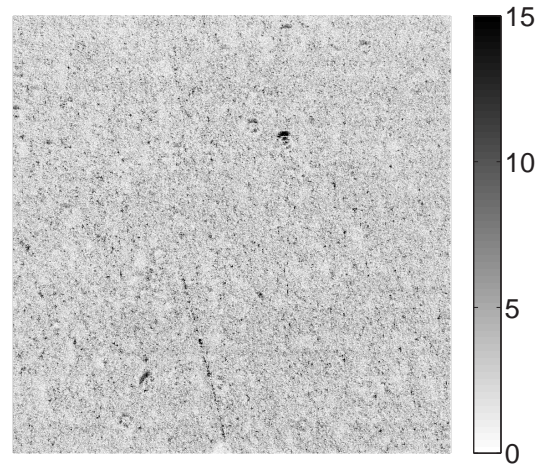

$-\mathrm{c}-$

Figure 12. Error map $\Phi$ for the $\mathrm{SiC}$ specimen for 32-pixels element using: no enrichment (a), discontinuous enrichment (b), discontinuous and singular enrichments (c). The analyzed pictures have Copyright (c) 2000 John Wiley \& Sons, Ltdan 8-bit digitization. Int. J. Numer. Meth. Engng 2000; 00:1-6 Prepared using nmeauth.cls 


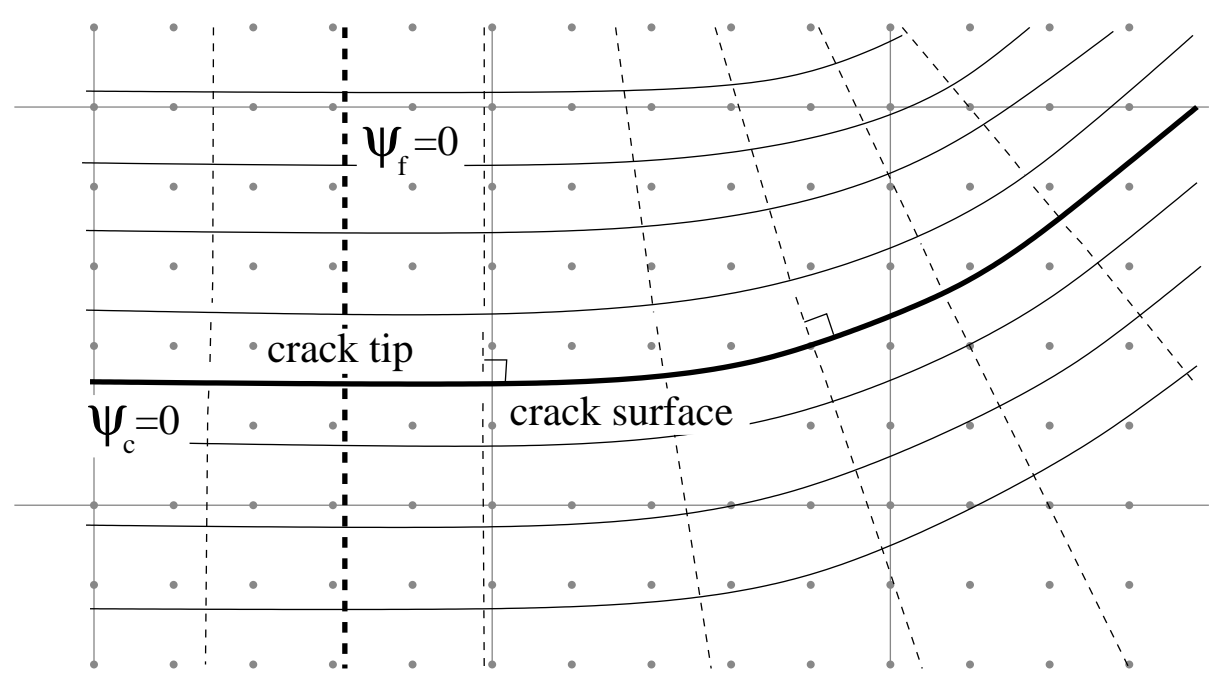

Figure 13. Schematic view of the crack description using level sets. $\psi_{f}$ is the front level set and $\psi_{c}$ the crack level set. Their intersection defines the crack tip and their normals are orthogonal. The gray lines depict the finite element mesh used for the displacement discretization and the gray dots the finite difference grid used for the level set discretization. 

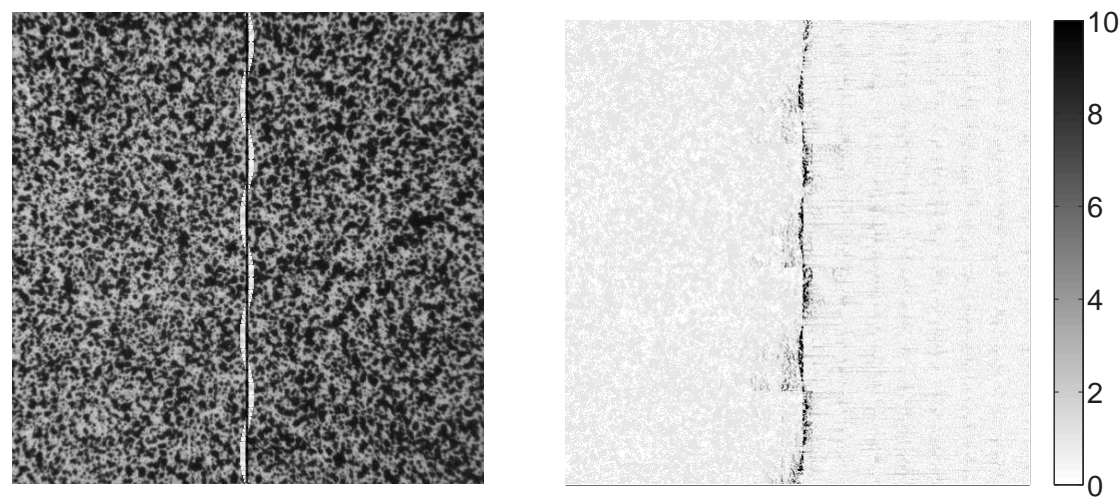

$-a-$
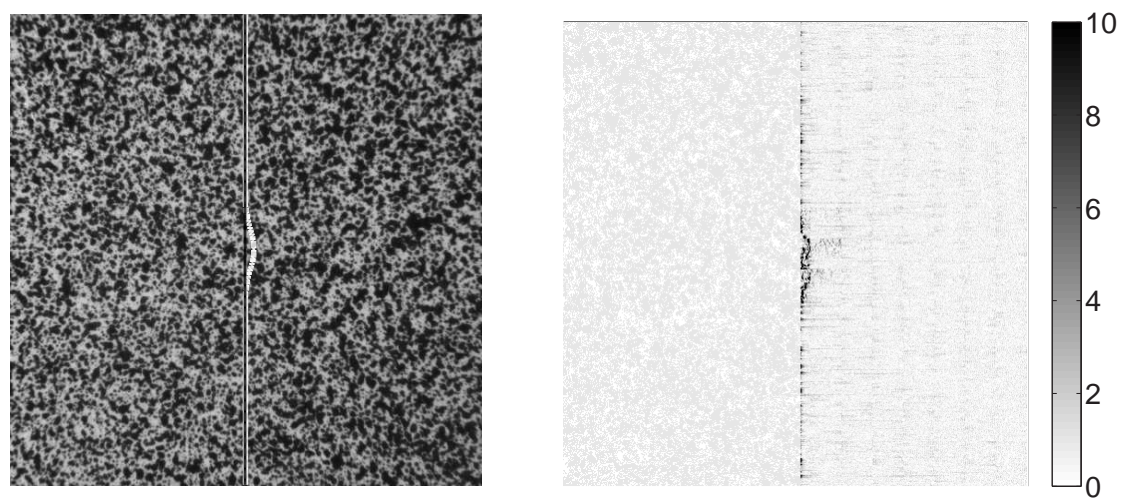

$-b-$

Figure 14. Left: Region of interest and discontinuity support. The gray line is the real discontinuity, the black line is the crack used for the calculation. Right: error maps $\Phi$ for artificial sinusoidal (a) and

Gaussian (b) discontinuities obtained with 32-pixel Q4 elements and discontinuous enrichment. 

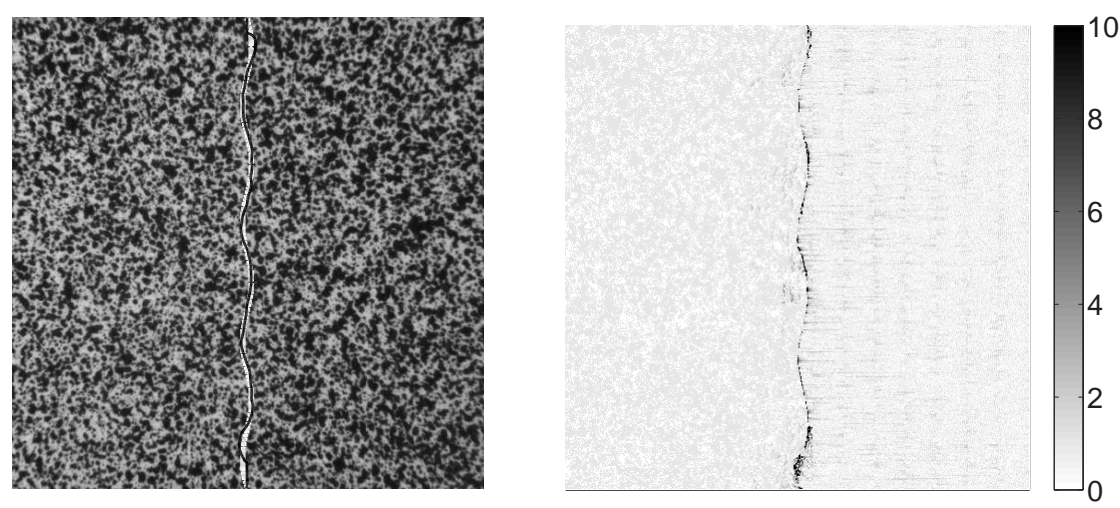

$-\mathrm{a}-$
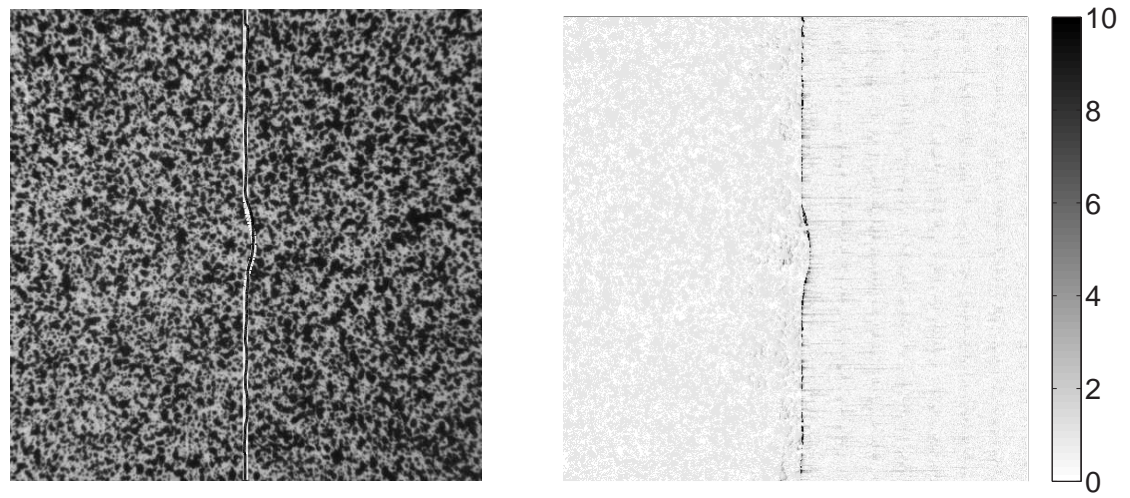

$-b-$

Figure 15. Left: Region of interest and discontinuity support. The gray line is the real discontinuity, the black line is the crack used for the calculation. Right: error maps $\Phi$ after crack shape optimization for artificial sinusoidal (a) and Gaussian (b) discontinuities obtained with 32-pixel Q4 elements and discontinuous enrichment. Because of the boundary effects on the edges of the image, the shape optimization is not performed inside the boundary elements. This is the reason why there is still a zone at the top and at the bottom wherein the error zone remains the same. 

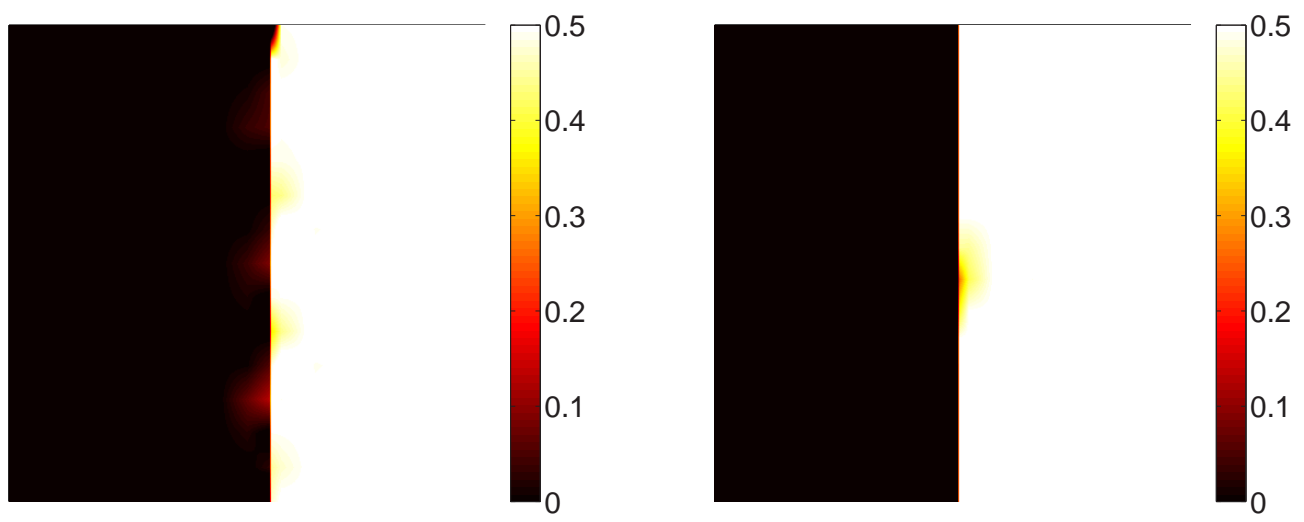

$-\mathrm{a}-$
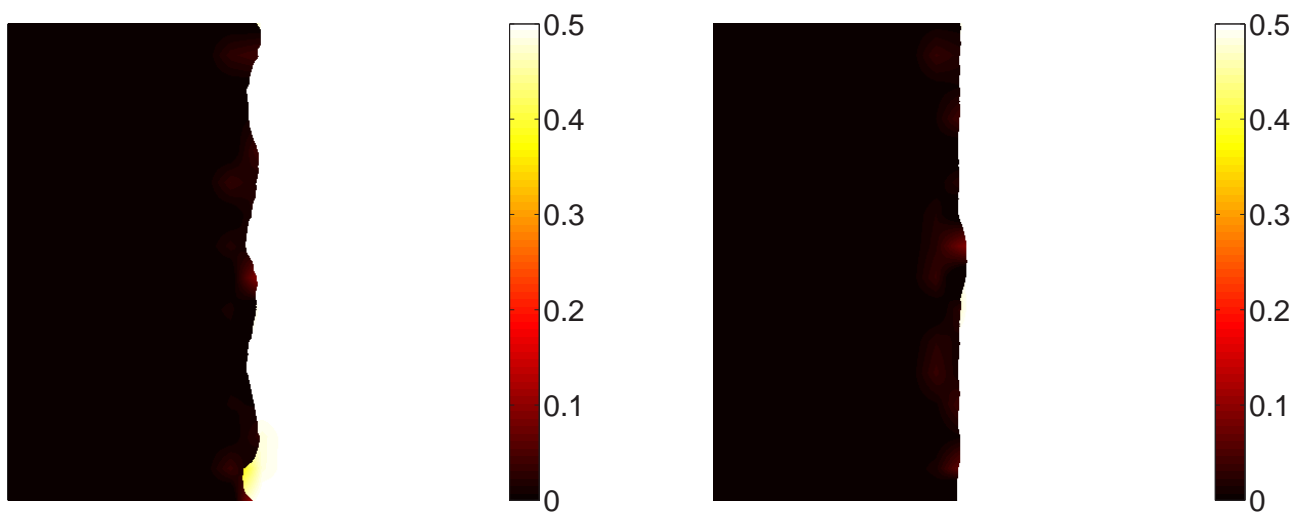

$-b-$

Figure 16. Horizontal displacement map for artificial sinusoidal and Gaussian discontinuities obtained with 32-pixel Q4 elements and discontinuous enrichment: -a-initial straight discontinuity, -b-optimized discontinuity profile. 


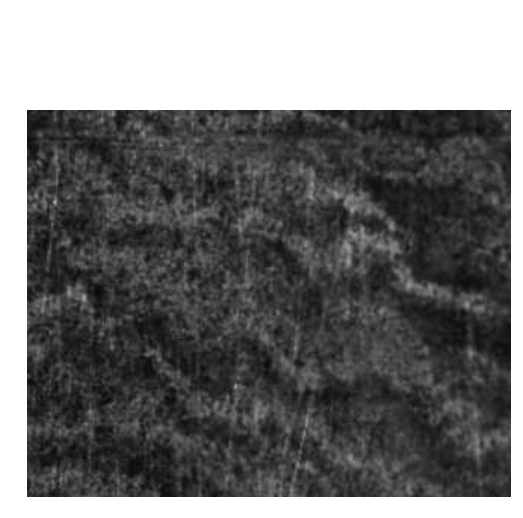

-a-

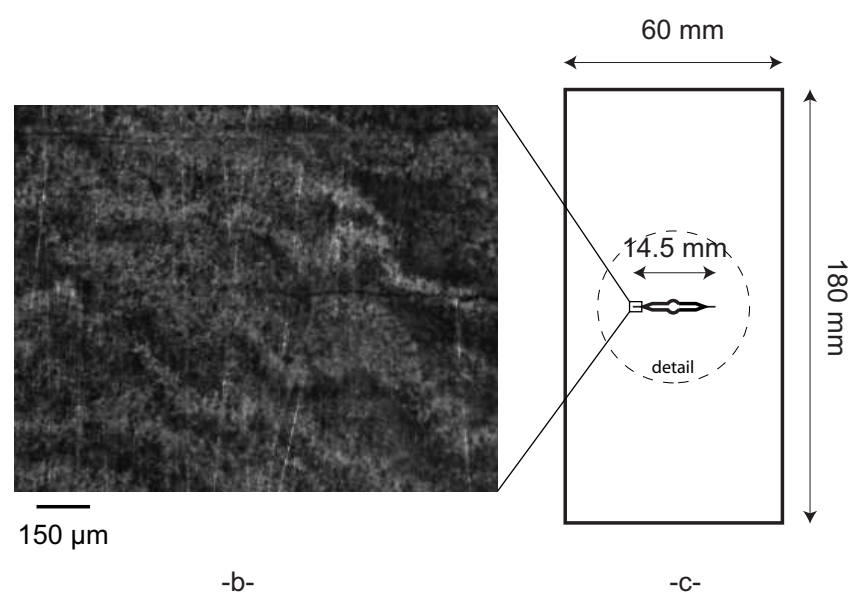

Figure 17. -a-Reference picture of the CCT test (16-bit digitization, $1024 \times 1280$ pixel resolution). -b-Picture with an open crack. -c-CCT geometry. 


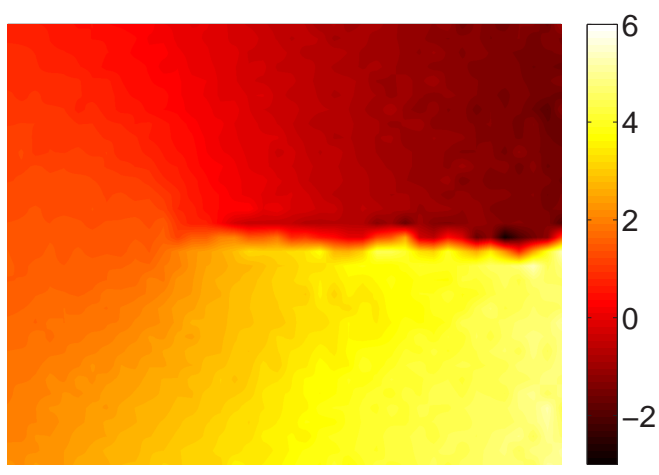

$-\mathrm{a}-$

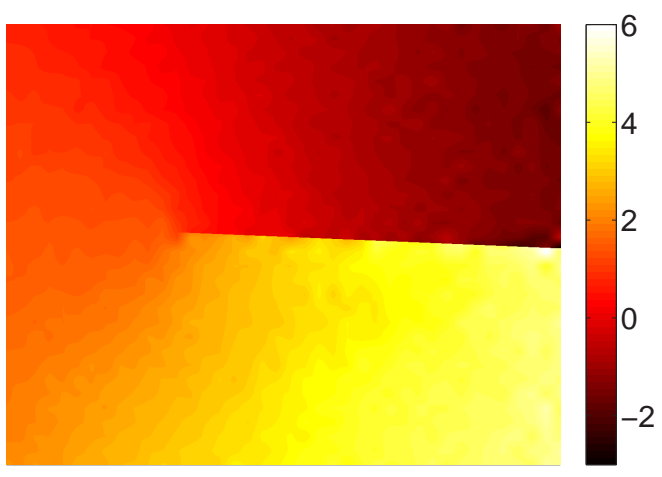

$-b-$

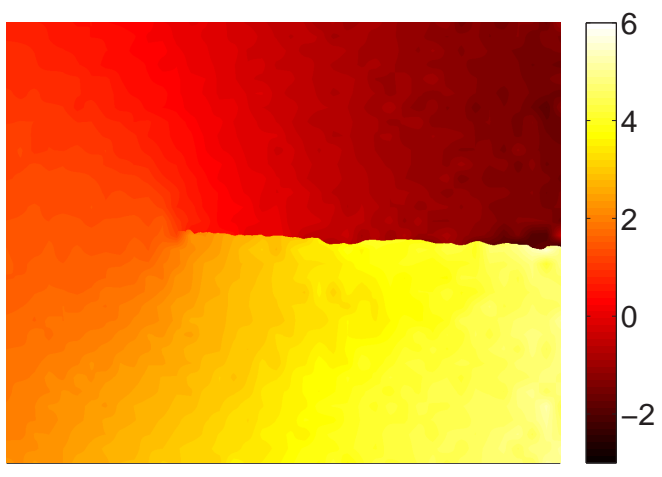

$-\mathrm{C}-$

Figure 18. Normal displacement map for the CCT specimen using: -a- 32-pixel elements with a standard Q4 interpolation, -b- 32-pixel elements with discontinuous enrichment, -c- same as top but using crack shape detection. The conversion factor is $p=2.08 \mu \mathrm{m} /$ pixel.

Copyright (c) 2000 John Wiley \& Sons, Ltd. $\quad$ Int. J. Numer. Meth. Engng 2000; 00:1-6

Prepared using nmeauth.cls 


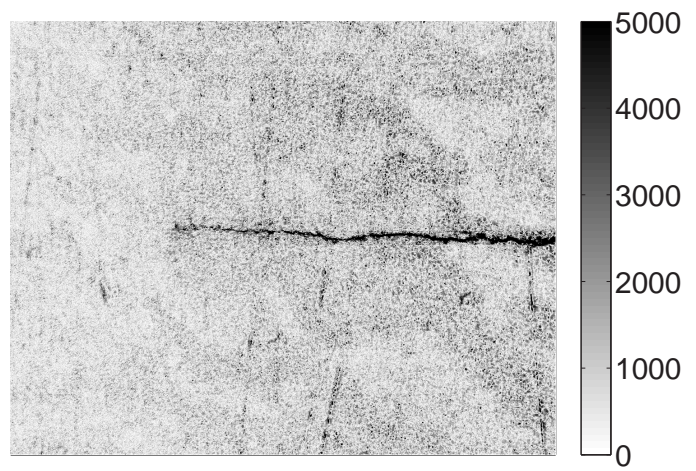

$-a-$

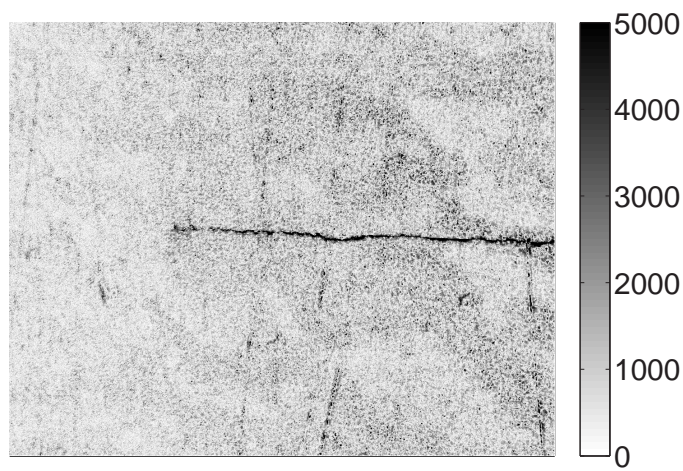

$-b-$

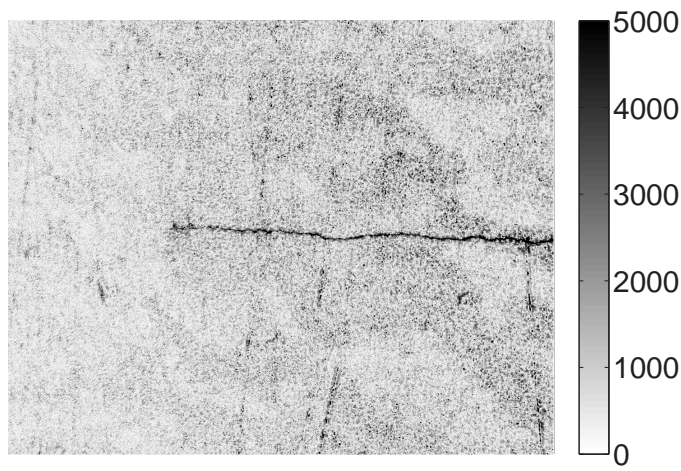

$-\mathrm{C}-$

Figure 19. Error map $\Phi$ in gray levels for the CCT specimen: -a-Q4 elements, -b-Q4 elements with discontinuous enrichment, -c-Q4 elements with discontinuous enrichment and crack shape detection.

Copyright (c) 2000 John Whe dynamic range of the analyzed pictures iss 16 bits 16 . Tumer. Meth. Engng 2000; 00:1-6 Prepared using nmeauth.cls 


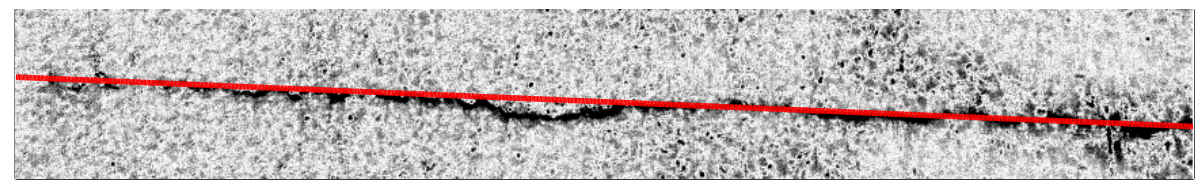

$-a-$

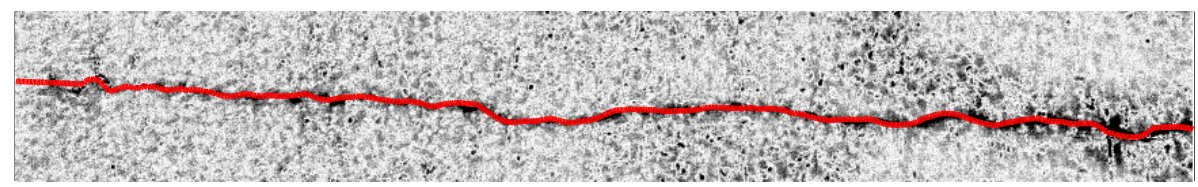

$-b-$

Figure 20. Straight (-a-) and optimized (-b-) crack path for the CCT specimen plotted on the error maps. The red line is the 0 -contour of the crack level set $\left(\psi_{c}\right)$ the error scale is the same as in Figure 19 and the size of the zone is $868 \times 124$ pixel. 


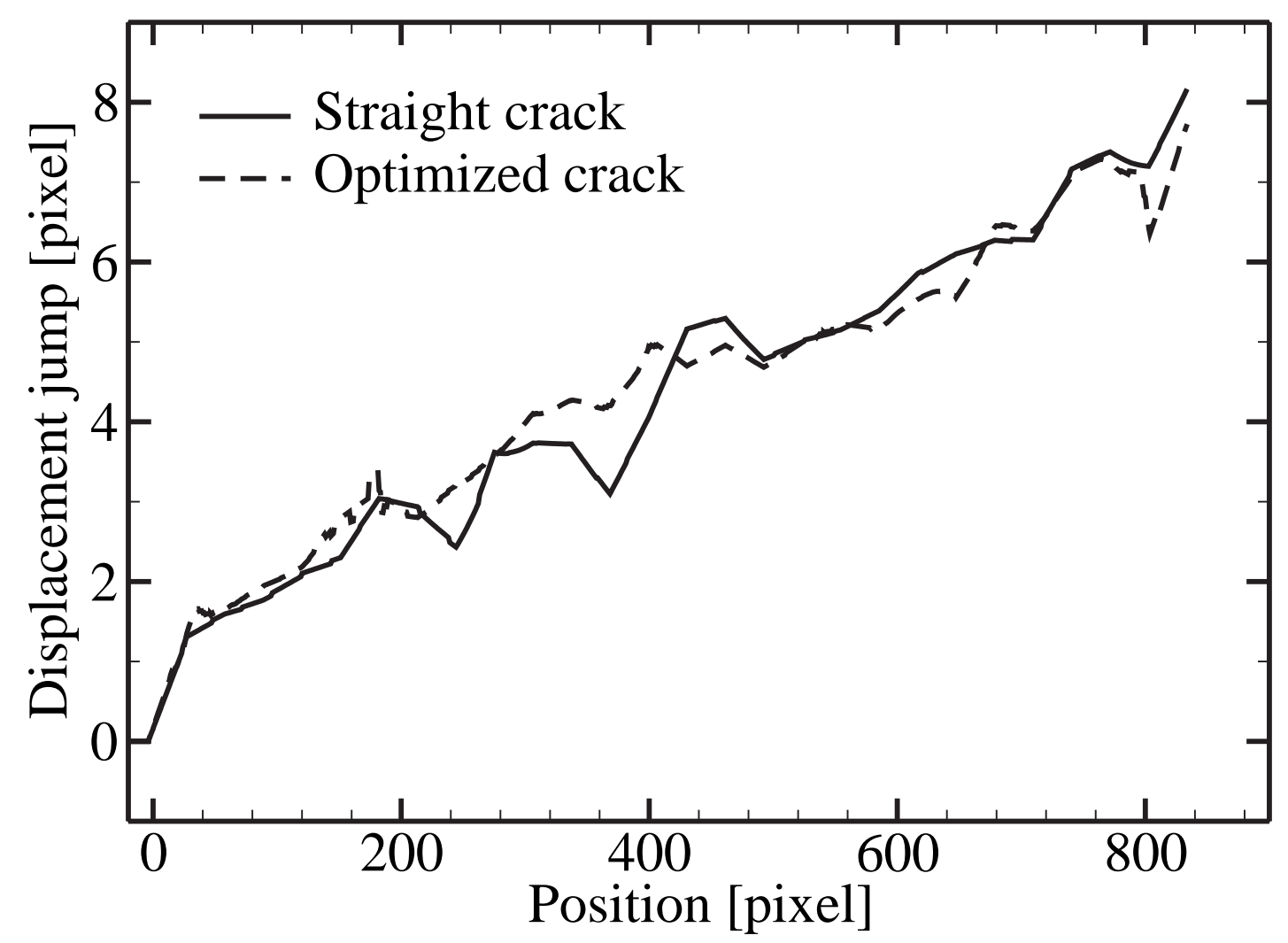

Figure 21. Crack opening displacement for 32-pixel elements using a discontinuous function and using discontinuous and tip functions obtained with the straight and the optimized cracks. 